Butoto Imani Wa RUSAATI, 2 Arusi Patience GENDUSA ${ }^{3}$ Sung-Hyun JoO ${ }^{1}$ Joo Won PARK ${ }^{1}$

Cephas Ndabaga MASUMBUKо ${ }^{4}$ Gentil Kaboyi IRAGI ${ }^{2}$

Koto-te-Nyiwa NgBoLUA

Astrid Matendo FURAHA ${ }^{6}$

Nkulu Kabange RoLLY, 8

Jun Won Kang ${ }^{1}$

${ }^{1}$ School of Forest Sciences and Land Architecture

College of Agriculture and Life Sciences Kyungpook National University

Daegu 41566

Republic of Korea

${ }^{2}$ Research Centre of Natural Sciences

(CRSN/Lwiro)

Department of Biology

DS Bukavu

Democratic Republic of Congo

${ }^{3}$ Independent Researcher

${ }^{4}$ Official University of Bukavu

Department of Biology

BP 570, Bukavu

Democratic Republic of Congo

${ }^{5}$ University of Kinshasa

Department of Biology

BP 127, Kinshasa XI

Democratic Republic of Congo

${ }^{6}$ Higher Institute of Rural Development of Bukavu

Department of Environnement and Sustainable Development

BP 2849, Bukavu

Democratic Republic of Congo

${ }^{7}$ Ministry of Agriculture

National Seed Service (SENASEM)

National Seed Laboratory

BP 904 KIN1, Kinshasa

Democratic Republic of Congo

${ }^{8}$ National Institute of Crop Science

Department of Southern Area Crop Science

RDA, Miryang 50424

Republic of Korea

Auteur correspondant /

Corresponding author:

Jun-Won KANG - jwkang15@knu.ac.kr

\section{A systematic review of antimalarial medicinal plants
in Democratic Republic of the Congo of antimalarial medicinal plants
in Democratic Republic of the Congo}

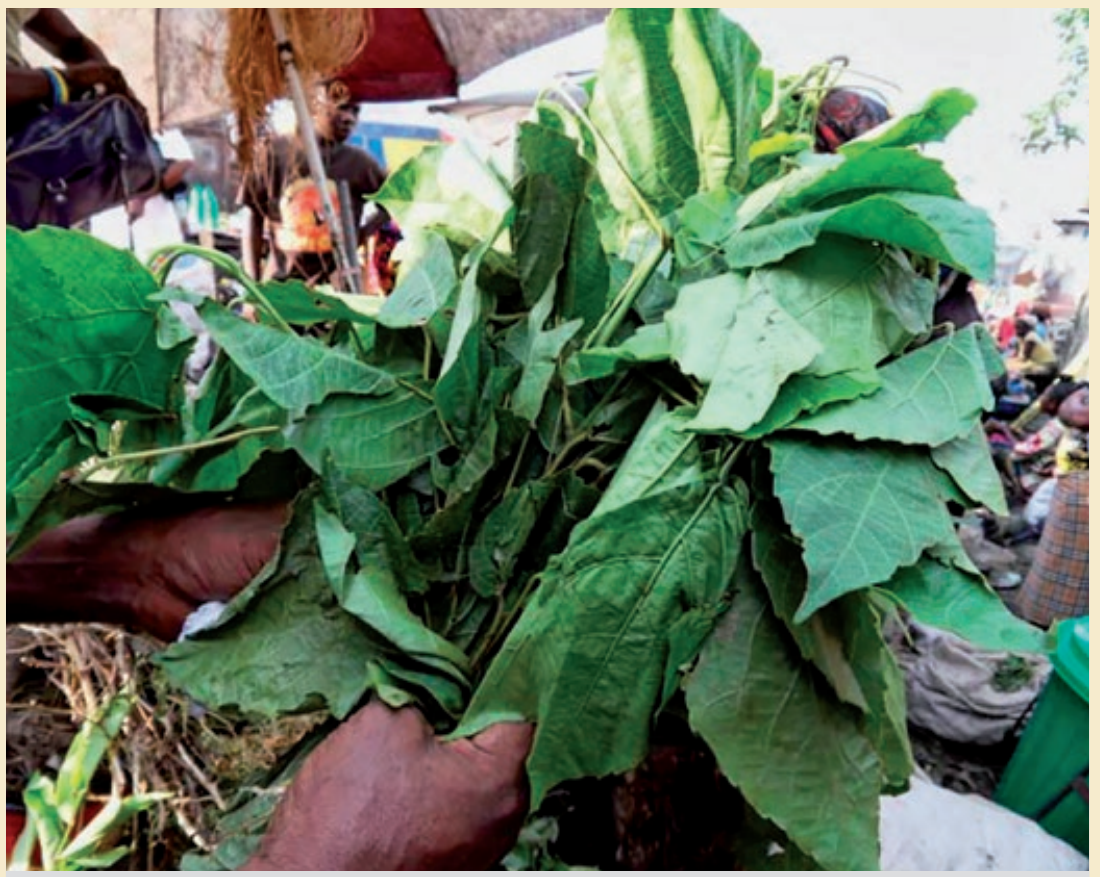

Photo 1.

Alchornea cordifolia, one of medicinal plants used to treat the symptoms of malaria in Democratic Republic of Congo.

Photo Konda et al. (2012).

Doi : 10.19182/bft2021.347.a31882 - Droit d'auteur @ 2021, Bois et Forêts des Tropiques - (c) Cirad - Date de soumission : 16 juin 2020 ; date d'acceptation : 16 décembre 2020 ; date de publication : 25 février 2021.

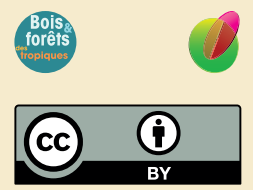

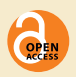

Licence Creative Commons :

Attribution - 4.0 International.

Attribution-4.0 International (CC BY 4.0)
Citer l'article / To cite the article

Rusaati B. I. W., Gendusa A. P., Joo S. H., Park J. W., Masumbuko N. C., Iragi K. G., Ngbolua K.-T.-N., Furaha M. A., Rolly N. K., Kang J. W., 2021. A systematic review of antimalarial medicinal plants in Democratic Republic of the Congo. Bois et Forêts des Tropiques, 347: 11-27. Doi : https://doi. org/10.19182/bft2021.347.a31882 


\section{RÉSUMÉ}

\section{Revue systématique des plantes médicinales antipaludiques utilisées en République démocratique du Congo}

En République démocratique du Congo (RDC), le paludisme est transmis par un parasite appelé Plasmodium falciparum. Le paludisme représente un problème majeur de santé publique dans ce pays en provoquant la mort de dizaines de milliers de personnes chaque année, en milieu urbain et rural. Des médicaments antipaludiques sont couramment utilisés mais des cas avérés de résistance à ces médicaments représentent un obstacle important à la lutte contre le paludisme. Il est donc impératif d'identifier de nouvelles molécules bioactives pouvant s'y substituer. De nombreuses plantes médicinales ayant des propriétés diverses s'utilisent en RDC pour traiter différentes maladies, dont le paludisme. Le présent article propose une revue systématique des ressources végétales antipaludiques en RDC. Sur la base de 28 articles sur l'ethnomédecine publiés entre 2001 et 2019, 232 espèces végétales appartenant à 67 familles de végétaux et identifiées dans 13 provinces ont été répertoriées pour le traitement du paludisme. De nombreuses espèces appartiennent aux familles des Fabacées, Astéracées, Euphorbiacées, Rubiacées et Apocyanacées. Les espèces dont l'utilisation est citée pour plus de trois provinces comprennent Cymbopogon citratus, Vernonia amygdalina, Rauvolfia vomitoria et Catharanthus roseus. La plupart des espèces identifiées comme plantes antipaludiques sont des essences ligneuses, principalement des phanérophytes. D'autre part, les principaux ingrédients identifiés pour la préparation des remèdes sont les feuilles, utilisées en décoction le plus souvent administrée par voie orale.

Mots-clés : traitement antipaludique, ethnomédecine, plantes médicinales, étude systématique, République démocratique du Congo.

\section{ABSTRACT}

\section{A systematic review of antimalarial medicinal plants in Democratic Republic of the Congo}

In Democratic Republic of the Congo (DRC), malaria is caused by a parasite called Plasmodium falciparum. Malaria is one of the country's major public health issues and responsible for the death of tens of thousands of people every year in both rural and urban environments. Antimalarial drugs are commonly used but some recorded cases of drug resistance are a major obstacle to controlling the spread of malaria. It is therefore essential to identify new bioactive molecules as an alternative. Many medicinal plants with different properties have been used as treatments for a variety of diseases in the DRC, including malaria. This study provides a systematic review of antimalarial plant resources in the DRC. From 28 papers on ethnomedicine published between 2001 and 2019, a total of 232 plant species belonging to 67 different plant families and identified in 13 provinces was reported in the treatment of malaria. A large number of these plant species belong to the Fabaceae, Asteraceae, Euphorbiaceae, Rubiaceae, and Apocyanaceae families. Species cited in more than three provinces include Cymbopogon citratus, Vernonia amygdalina, Rauvolfia vomitoria and Catharanthus roseus. Most of the species identified as antimalarial plants were tree species, with phanerophytes predominating. In addition, leaves were identified as the main ingredients for preparing remedies, most commonly by decoction administered orally.

Keywords: antimalarial treatment, ethnomedicine, medicinal plants, systematic review, Democratic Republic of the Congo.

\section{RESUMEN}

\section{Revisión sistemática de las plantas medicinales antipalúdicas de la República Democrática del Congo}

En la República Democrática del Congo (RDC) el paludismo está causado por un parásito llamado Plasmodium falciparum. El paludismo es uno de los principales problemas de salud pública del país y es responsable de la muerte de decenas de miles de personas cada año, tanto en entornos rurales como urbanos. Habitualmente se utilizan fármacos antipalúdicos, aunque se registran casos de resistencia a los medicamentos, que son un obstáculo importante para controlar la propagación de la malaria. Por lo tanto, es esencial identificar nuevas moléculas bioactivas como alternativa. En la RDC se han utilizado muchas plantas medicinales con diferentes propiedades como tratamiento de diversas enfermedades, entre ellas la malaria. Este estudio realiza una revisión sistemática de los recursos vegetales antipalúdicos en la RDC. En 28 artículos sobre etnomedicina publicados entre 2001 y 2019, un total de 232 especies de plantas para el tratamiento de la malaria pertenecientes a 67 familias diferentes se identificaron en 13 provincias. Un gran número de estas especies vegetales pertenecen a las familias Fabaceae, Asteraceae, Euphorbiaceae, Rubiaceae y Apocyanaceae. Las especies citadas en más de tres provincias incluyen Cymbopogon citratus, Vernonia amygdalina, Rauvolfia vomitoria y Catharanthus roseus. La mayor parte de las plantas antipalúdicas identificadas son especies arbóreas, con predominio de las fanerófitas. $Y$ las hojas son los principales ingredientes para la preparación de curas, mayoritariamente por decocción administrada por vía oral.

Palabras clave: tratamiento antipalúdico, etnomedicina, plantas medicinales, revisión sistemática, República Democrática del Congo. 


\section{Introduction}

Malaria, a disease caused by the parasite Plasmodium falciparum, is one of the major public health problems in many tropical countries. This disease is spread by mosquitoes (Landis et al., 2009; Messina et al., 2011). According to the World Health Organization (WHO), about 228 million cases and more than 405 thousand deaths related to malaria have occurred in 2018 around the world, with the majority of deaths (93\%) found in Africa (WHO, 2019).

In Democratic Republic of the Congo (DRC), reports indicated that, despite the efforts to prevent the spread of malaria, and alleviate its detrimental effects on the people's health nationwide (Swana et al., 2018), other forms of challenges, such as drug resistance of the pathogen was identified as obstacle to control efficiently the spread of the disease in the sub-Saharan Africa (Alker et al., 2008; Mobula et al., 2009; Mvumbi et al., 2015). To address this situation, there is a strong necessity to identify novel substances or bioactive molecules having the potential to overcome the drug resistance of the Plasmodium falciparum, eventually due to the recurrent use of similar molecules. Thus, medicinal plants could serve as an alternative to achieve this goal (Silva et al., 2011; Ntie-Kang et al., 2014), since they are relatively cost-effective and highly accessible (Madureira et al., 2002; Muganga et al., 2010).

The benefit of plants as bio-resources and their potentialities to treat different diseases has traditionally been highlighted by several research (Arshad et al., 2014; Zarei et al., 2017), and it is considered essential for human health care (Asadi-Samani et al., 2013). The worldwide use and distribution of bio-resources enclose a large potential to unveil the undescribed aspects of medicinal plants, yet undiscovered (Balima et al., 2018).

According to the $\mathrm{WHO}$, around $80 \%$ of the population living in developing countries rely on traditional medicine for treating diseases (Kamatenesi-Mugisha and Oryem-Origa, 2005; Mahomoodally, 2013). Moreover, the extensive use of traditional medicines in Africa could be associated to the history and culture or customs, and economic environment (Mahomoodally, 2013). Medicinal plants still represent an important source of medical treatment in developing countries (Tabuti et al., 2003).

The Congo Basin region itself is megadiverse in plant species, including medicinal plants (Light et al., 2016). Screening studies for antimalarial medicinal plant species from Cameroon (Betti, 2002; Saotoing et al., 2011; Titanji et al., 2008; Kuete and Efferth, 2010; Betti et al., 2013a), Gabon (Betti et al., 2013b), the Central African Republic (Lakouéténé et al., 2009), Republic of Congo (Mbatchi et al., 2006; Nsonde-Ntandou et al., 2005) and Guinea Equatorial (Gomez Marín and Merino Cristóbal, 1990) have demonstrated the potential of traditional medicinal plants as source of antimalarial substances. Furthermore, the DRC, with about half of the African humid forests, ranks fifth in the world for the diversity of plant species (UNESCO, 2010; Masunda et al., 2019). It is estimated that the flora of the DRC comprises 377 families, 2,196 genera, and over

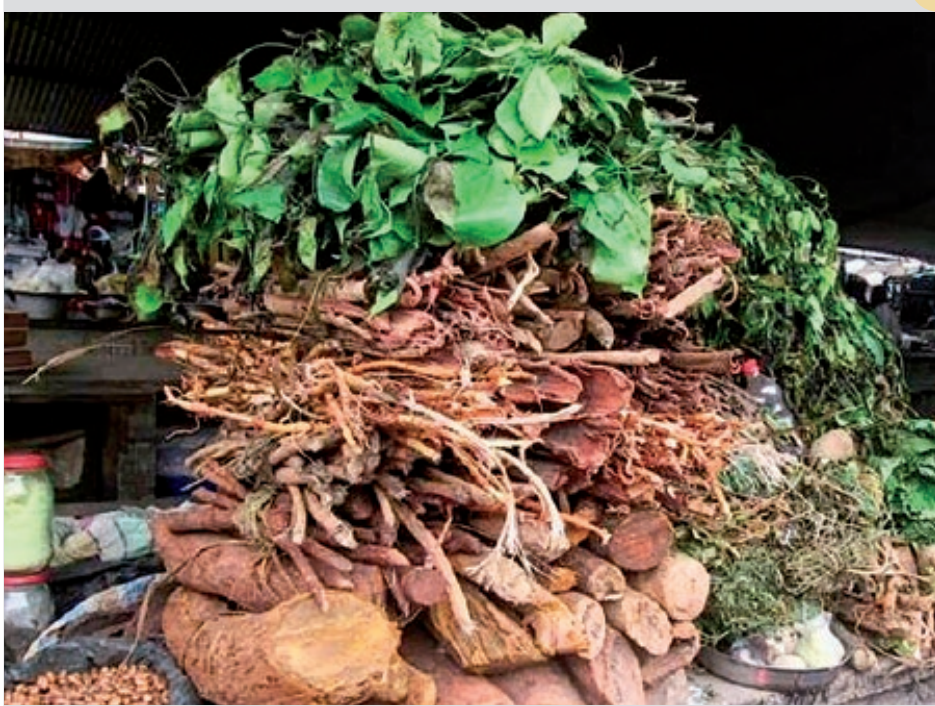

Photo 2.

Illustration of a variety of medicinal plants exposed

in a local market in Kinshasa.

Photo K.-T.-N. Ngbolua et al. (2016).

11,000 species (Mbala, 2003). Many plants species in the DRC have been studied and described for their beneficial impact for treating various diseases in numerous scientific papers. Thus, the purpose of this study is to develop a systematic review of scientific papers on ethnomedicine, particularly on the treatment of malaria.

\section{Methods}

\section{Sources of information}

Through literature review and data mining, previous published papers related to the field of ethnobotanyof medicinal plants in the DRC, were collected during December 2019, using available internet browsers.

The research was extended to the identification of references listed in retrieved articles. The major key words employed during the search, either in French or in English, were: "ethno-medicinal plants in DR of Congo" OR "ethnobotanical study in DR of Congo" OR "ethno pharmacology in DR of Congo" OR "phyto therapy in DR of Congo" OR "herbal treatments in DR of Congo" OR "anti-malaria plants in DR of Congo". In addition to the published scientific papers on the subjects of interest, all information found to be necessary for the study were included as well, collected from scientific works, such as books, academic theses (Masters and doctorate thesis dissertations), while considering the year of publication, the methodology, or the study language.

\section{Analysis}




\section{Results}

\section{Selected antimalarial related studies}

In the perspective of using only studies providing useful data and information related to the use of medicinal plants as a treatment for malaria, an initial selection was performed. As a result, all studies with no relevant data on antimalarial plants were discarded, and only 41 full-text studies were evaluated in order to verify the evidence of the antimalarial plants. In addition, another 13 studies falling either into the category of review article or in vitro studies were excluded. Consequently, after the screening and selection process, 28 publications and academic reports (Masters and Doctorate Theses) were retained for further investigations (figure 1). Of this number, the earliest article was published in 2001 but about $92.9 \%$ of the publications were released between 2010-2019. Studies were conducted on 13 different provinces in the DRC as illustrated in figure 2. Moreover, the majority of plant species (185 species, $79.7 \%$ ) was reported to be used in at least one province, and 27 species (11.6\%) were listed in at least two provinces (table I). Meanwhile, 20 species (8.6\%) were mentioned in more than two provinces. An illustration of the top 20 plant species widely distributed across the country (used in more than 2 provinces) is given in table II.

(ii) mode of preparation: decoction, maceration, infusion, grinding, expression, extorsion, incineration, ash, etc.; (iii) way of administration: oral, enema, inhalation, bath, friction, chewing, tattoo, instillation; and (iv) the geographical location of the study. In the case of unavailability of data in one of the above groups, the corresponding field was marked "NR (not referenced)".

The dataset was taxonomically standardized (synonym and misspelling) and updated following guidelines in the online websites: the plant list ${ }^{1}$ or/and African plant database $^{2}$. The Angiosperm Phylogeny Group (APG III) was used to revise and update the family names (APG, 2009). The antimalarial plants species were characterized by morphology types: tree, shrub, sub-shrub, liana, herb. The life-form analysis focused on 223 species and was based on Raunkiaer's system: Phanerophytes, Chamæphytes, Hemicryptophytes, Geophytes or Cryptophytes, Therophytes, Aerophytes and Epiphytes.

The conservation status of each anti-malaria species was determined by the IUCN red list of threatened species (IUCN, 2019) and includes: Not Evaluated (NE), Data Deficient (DD), Least concern (LC), Near threatened (NT), Vulnerable (VU), Endangered (EN), Critically endangered (CR), Extinct in the Wild (EW) and Extinct (EX).

\section{1 http://www.theplantlist.org \\ 2 http://www.ville-ge.ch}

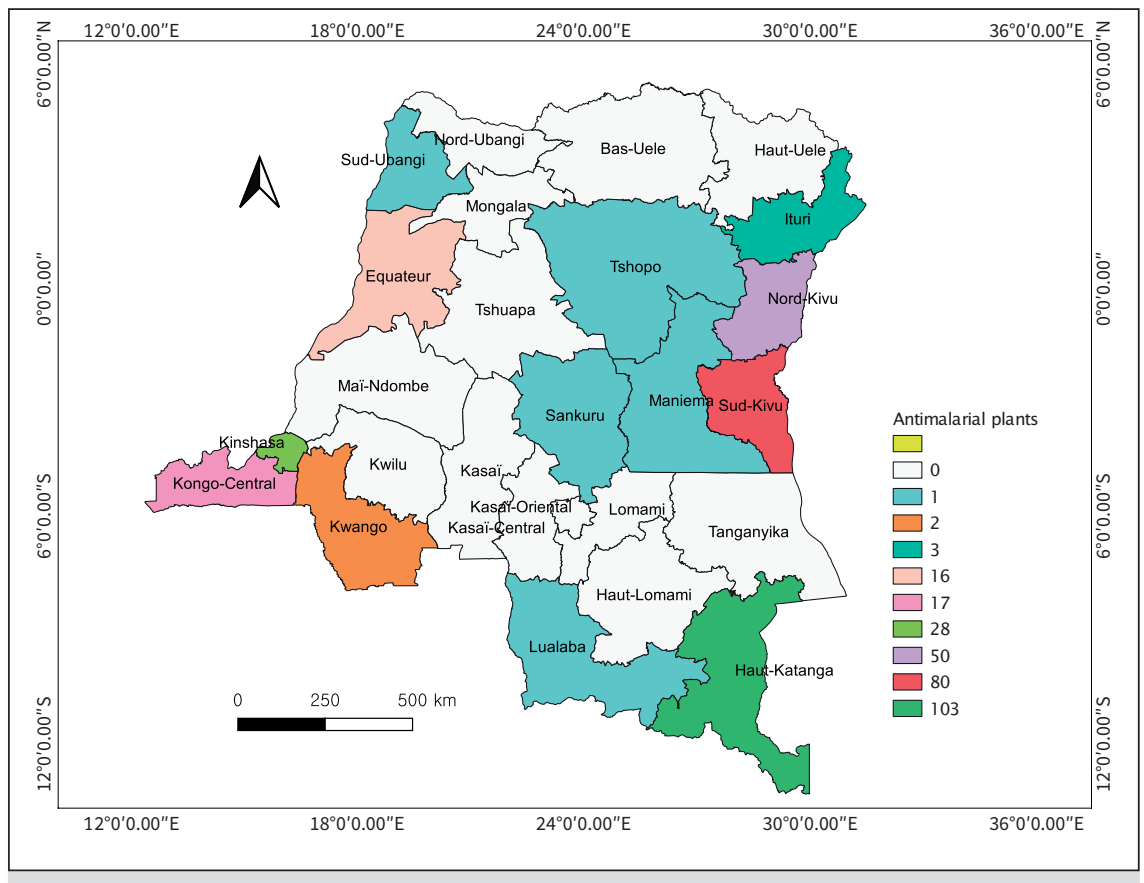

Figure 2.

Map of the spatial distribution of studies related to antimalaria treatment using plant species in the Democratic Republic of Congo. The highlighted zones on the map indicate the studies area related to antimalaria treatment using medicinal plants species. The color scheme in the legend shows the number of studies in each province. 
Table I.

Level of antimalarial plants richness within each province.

\begin{tabular}{|c|c|c|c|c|c|c|c|c|c|c|c|c|c|}
\hline Provinces & 1 & 2 & 3 & 4 & 5 & 6 & 7 & 8 & 9 & 10 & 11 & 12 & 13 \\
\hline Species richness & 103 & 80 & 50 & 28 & 17 & 16 & 3 & 2 & 1 & 1 & 1 & 1 & 1 \\
\hline $\begin{array}{l}\text { Specific species } \\
\text { in the province }\end{array}$ & 82 & 48 & 17 & 19 & 9 & 7 & 1 & 0 & 1 & 0 & 1 & 0 & 0 \\
\hline $\begin{array}{l}\text { Species shared by } \\
\text { other provinces }\end{array}$ & 21 & 32 & 33 & 9 & 8 & 9 & 2 & 2 & 0 & 1 & 0 & 1 & 1 \\
\hline
\end{tabular}

Provinces: 1: Haut-Katanga, 2: Sud-Kivu, 3: Nord-Kivu, 4: Kinshasa, 5: Kongo-Central, 6: Equateur, 7: Ituri, 8: Kwango, 9: Tshopo, 10: Sud-Ubangi, 11: Lualaba, 12: Sankuru, 13: Maniema.

\begin{tabular}{|c|c|c|}
\hline \multicolumn{3}{|c|}{$\begin{array}{l}\text { Table II. } \\
\text { List of the most distributed antimalarial plants. }\end{array}$} \\
\hline Species & \multirow{2}{*}{$\begin{array}{c}\text { Number of } \\
\text { provinces } \\
5\end{array}$} & \multirow{2}{*}{$\begin{array}{l}\text { Provinces } \\
1,2,3,5,6\end{array}$} \\
\hline Senna occidentalis & & \\
\hline Cymbopogon citratus & 5 & $1,2,3,4$ \\
\hline Vernonia amygdalina & 4 & $1,2,3$ \\
\hline Rauvolfia vomitoria & 4 & $1,3,4,6$ \\
\hline Catharanthus roseus & 4 & $1,2,3,5$ \\
\hline Carica papaya & 3 & $1,2,3$ \\
\hline Bidens pilosa & 3 & $1,2,3$ \\
\hline Alstonia boonei & 3 & $4,7,10$ \\
\hline Arachis hypogaea & 3 & $2,3,5$ \\
\hline Citrus limon & 3 & $1,2,3$ \\
\hline Erythrina abyssinica & 3 & $1,2,3$ \\
\hline Eucalyptus globulus & 3 & $1,2,3$ \\
\hline Harungana madagascariensis & 3 & $1,2,6$ \\
\hline Jatropha curcas & 3 & $1,5,8$ \\
\hline Lantana camara & 3 & $1,2,3$ \\
\hline Morinda morindoides & 3 & $4,5,6$ \\
\hline Persea americana & 3 & $2,3,6$ \\
\hline Physalis peruvianna & 3 & $1,2,3$ \\
\hline Psidium guajava & 3 & $1,2,3$ \\
\hline Tithonia diversifolia & 3 & $1,2,3$ \\
\hline \multicolumn{3}{|c|}{$\begin{array}{l}\text { Provinces: 1: Haut-Katanga, 2: Sud-Kivu, 3: Nord-Kivu, } \\
\text { 4: Kinshasa, 5: Kongo-Central, 6: Equateur, 7: Ituri, } \\
\text { 8: Kwango, 9: Tshopo, 10: Sud-Ubangi, 11: Lualaba, } \\
\text { 12: Sankuru, 13: Maniema. }\end{array}$} \\
\hline
\end{tabular}

$82 \%$ are dicots, and nearly $14 \%$ are monocots, and only $3 \%$ are gymnosperms and pteridophytes. The dicotyledonous group is represented by 55 families and 169 genera, while the monocotyledonous group is represented by 10 families and 10 genera. The investigation on the life-form of the antimalarial plants showed that Phanerophytes represent $68.3 \%$ of the species cited, followed by Therophytes (13.4\%), Chamæphytes (12.9\%), Geophytes (4.5\%), and Hemycryptophytes (0.9\%) (table III). Regarding the morphological type, figure 3 shows that woody plants (66.8\%) were the dominant type (trees: $38.8 \%$, shrubs: $16.8 \%$, sub-shrub: $3.02 \%$, and liana: $8.2 \%$ ). About half of the reported antimalarial plant species across the country (48.3\%) belong to the following families: Fabaceae (30 species), Asteraceae (27 species), Rubiaceae (15 species), Euphorbiaceae (14 species), Apocynceae (10 species), Annonaceae (8 species) and Meliaceae (8 species). Around 49.2\% of the families contributed with only one species to the antimalarial plant species, while 145 genera are represented by a single antimalarial plant species. Euphorbia (Euphorbiaceae) and Strychnos (Loganiaceae) were represented by 4 plant species each. In addition to Euphorbia and Strychnos, Acacia, Afromomum, Aloe, Chenopodium, Combretum, Dalbergia, Ficus, Landolphia, Morinda, Senna and Ziziphus have 3 species each (appendix 1).

\section{Parts used, mode of preparation, and route of administration}

The utilization of plant parts and their mode of preparation is subjected to their accessibility and the knowledge of indigenous people (Umair et al., 2019). The results of the analysis of the plant parts used

\section{Diversity and morphology of antimalarial plants}

A total of 232 species of medicinal plants, distributed in 181 genera, and belonging to 67 families were mentioned as being involved in the treatment of malaria in different parts of the DRC (figure 2; table III). Among these families, about show that the leaves were the most used parts of plant by traditional healers, which accounted for about $60 \%$ of the total parts of plants used, followed by roots (32.7\%), and stems/ bark (22.4\%). A few plants were harvested for their fruits or seeds/grains $(6.5 \%)$ or used whole $(5.2 \%)$. In $3.4 \%$ of cases, the parts of the plant used were not referenced (figure 4). 
Table III.

Summary of the antimalarial plants used in Democratic Republic of Congo.

\begin{tabular}{|c|c|c|c|c|c|}
\hline \multirow[b]{3}{*}{ Family } & \multirow{3}{*}{$\begin{array}{c}\text { Pteridophytes } \\
1\end{array}$} & \multirow{3}{*}{$\begin{array}{c}\text { Gymnosperms } \\
1\end{array}$} & \multicolumn{2}{|c|}{ Angiosperms } & \multirow{3}{*}{$\begin{array}{r}\text { Total } \\
\\
67\end{array}$} \\
\hline & & & Dicotyledons & Monocotyledons & \\
\hline & & & 55 & 10 & \\
\hline Genus & 1 & 1 & 169 & 10 & 181 \\
\hline Species & 1 & 1 & 211 & 19 & 232 \\
\hline Phanerophyte & - & 1 & 148 & 4 & 153 \\
\hline Chamaephyte & - & 0 & 25 & 3 & 28 \\
\hline Hemycryptophyte & - & 0 & 2 & 0 & 2 \\
\hline Therophyte & - & 0 & 28 & 2 & 30 \\
\hline Geophyte & - & 0 & 0 & 10 & 10 \\
\hline
\end{tabular}

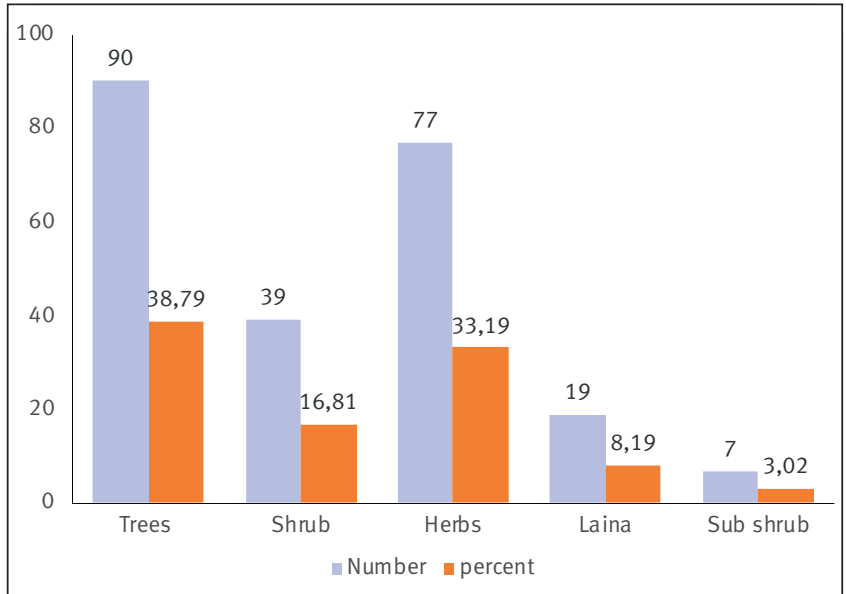

Figure 3.

Morphology of the most cited antimalarial plants species.

Sixteen methods were used to prepare plant materials. Among these, the majority used decoction (169 reported), followed by maceration (48), infusion (27), grinding and sap (4 each), powder (3) expression, extorsion, and spray (2 each); chewing, ash, incineration, milling, paste, pounding, and leaves roasted in palm oil (1 each); and 19 were not referenced (NR) (figure 5). The majority (156) of antimalarial remedies were taken orally, followed by enema/anal/suppository (12), bath, inhalation, and instillation (7 each). In rare instances, treatment was administered by rubbing leaves all over the body (2) and by tattoo (1). Sixty-five reports failed to indicate the mode of administration of the plant medicines.

\section{Threat status}

The unsustainable exploitation of plant species has resulted in a high vulnerability for certain species (Raj et al., 2018). It was found that 49 plant species used as a remedy for treating malaria in the DRC were reported in the IUCN's Red List (table IV). According to the IUCN list, one species was

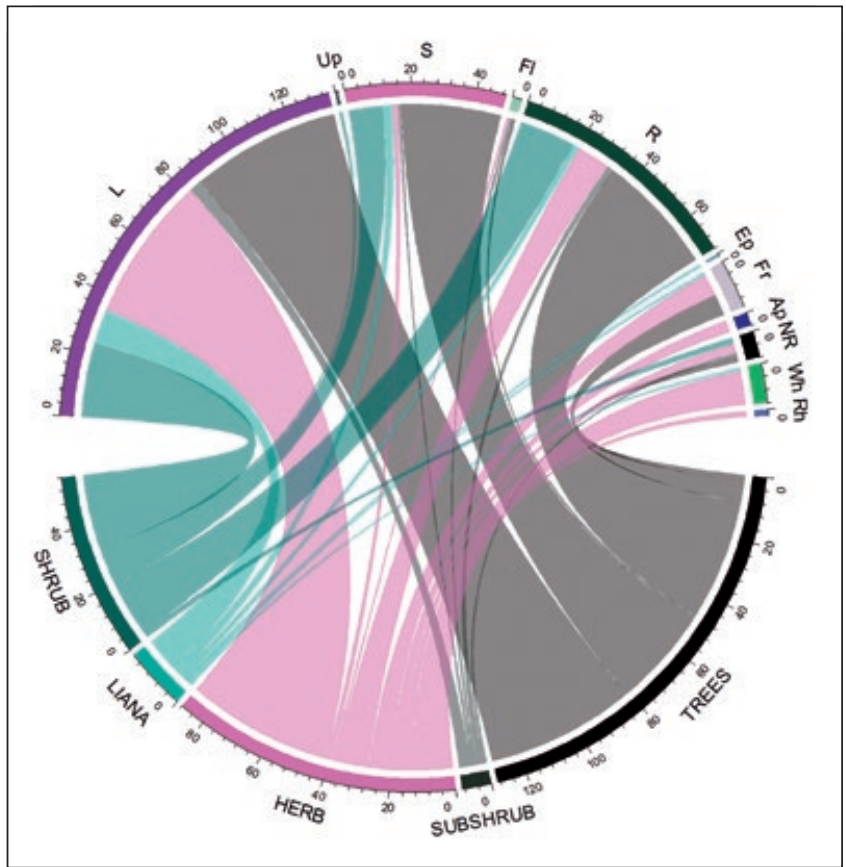

Figure 4.

Distribution of plant parts commonly used in ethnomedicine by morphological types. Eleven parts used (top half of circle) include (left to right): leaves (L), underground part (Up), stems (S), flower (Fl), roots (R), external part (Ep), fruit, seed, grains (Fr), aerial part (Ap), not recorded (NR), whole plant (Wh), and rhizomes (Rh). Morphological types (bottom half of circle). Scale numbers around the circle indicate cited time.

classified as "data deficient”, 43 as "least concern", three as "vulnerable", one as "near threatened", and one as "critically endangered". The other species were not included yet in the list. The international trade in a few of the species studied is also banned by the CITES treaty ${ }^{3}$ : Aloe christianii, Aloe dawei, Dalbergia boehmii, Dalbergia chapelieri, Dalbergia nitidula, Euphorbia ingens, Euphorbia tirucalli, and Prunus africana.

\footnotetext{
${ }^{3}$ www.cites.org
} 


\section{Table IV.}

The conservation status of some medicinal plants used to treat malaria in the Democratic Republic of Congo.

\section{Species}

Autranella congolensis

Dalbergia chapelieri

Lebrunia buchaie, Prunus africana, Pseudospondias microcarpa

Annona senegalensis, Annickia chlorantha, Azadirachta indica, Bobgunnia madagascariensis,

Cassia alata, Cassia sieberiana, Carapa procera, Combretum molle, Combretum zeyheri, Eucalyptus

citriodora, Eucalyptus globulus, Euphorbia ingens, Euphorbia tirucalli, Erythrina abyssinica, Ficus

exasperate, Ficus thonningii, Harungana madagascariensis, Hymenocardia acida, Isoberlinia

angolensis, Isolona hexaloba, Julbernardia paniculata, Melia azedarach, Monodora laurentii,

Monodora myristica, Ochna schweinfurthiana, Parinari curatellifolia, Parkia zenkeri, Pentaclethra

macrophylla, Pericopsis angolensis, Persea americana, Piper capense, Pterocarpus angolensis,

Pterocarpus tinctorius, Ranunculus multifidus, Raphia gentiliana, Rauvolfia caffra, Terminalia mollis,

Thomandersia hensii, Spathodea campanulata, Syzygium guineense, Xylopia aethiopica, Ziziphus

abyssinica, Ziziphus mucronata

Mangifera indica

\section{Status}

Critically endangered

Near threatened

Vulnerable

Least concern

Data deficient

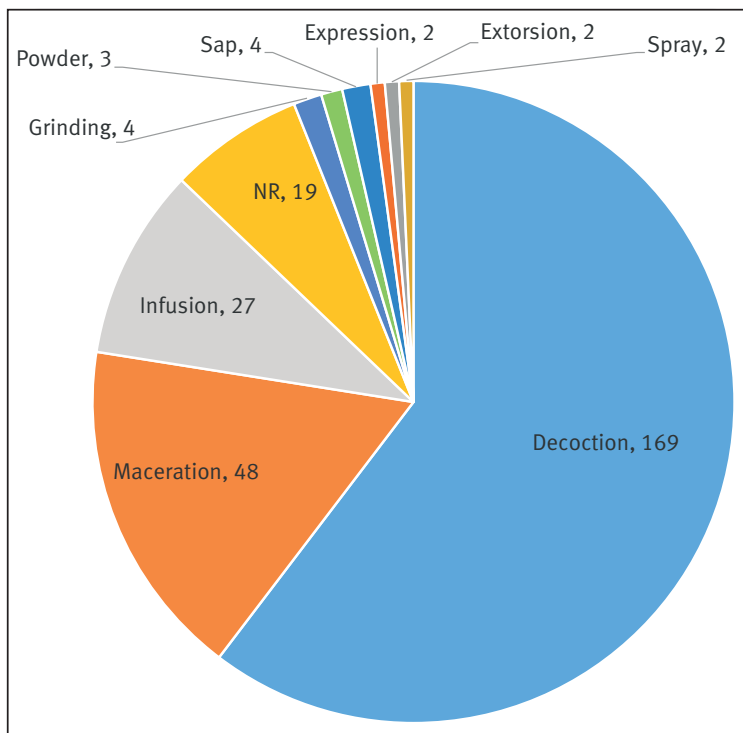

Figure 5.

Preparation methods of the most cited antimalarial plants.

\section{Discussion}

Two hundred thirty-two medicinal plants associated with the treatment of malaria in DRC that distributed across 181 genera and 67 families were identified. From these families, Fabaceae, Asteraceae, Euphorbiaceae, and Rubiaceae had the highest number of antimalarial plants species. These findings are similar to those obtained by Asase et al. (2010), Traore et al. (2013), and Taek et al. (2018) who reported that the Fabaceae had a high number of antimalarial plants species compared to other families. In a converse approach, Iyamah and Idu (2015) indicated that Fabaceae counts the highest number of antimalarial plants in Southern Nigeria, followed by Asteraceae. The predominance of medicinal plants from Fabaceae, Asteraceae ad Rubia-

\section{Table V.}

The number of species (Ndjele, 1988) and the number of antimalarial plants per family in the terrestrial flora of the Democratic Republic of Congo.

\begin{tabular}{|l|c|c|c|}
\hline Families & $\begin{array}{c}\text { Democratic Republic } \\
\text { of Congo }\end{array}$ & $\begin{array}{c}\text { Antimalarial } \\
\text { plants }\end{array}$ & Rank \\
\hline Fabaceae & 893 & 30 & 1 \\
\hline Asteraceae & 729 & 27 & 2 \\
\hline Rubiaceae & 674 & 15 & 3 \\
\hline Euphorbiaceae & 377 & 14 & 4 \\
\hline Apocynaceae & 187 & 10 & 5 \\
\hline Annonaceae & 119 & 8 & 6 \\
\hline Meliaceae & 47 & 8 & 6 \\
\hline Lamiaceae & 307 & 6 & 7 \\
\hline Myrtaceae & 31 & 6 & 7 \\
\hline Solanaceae & 96 & 6 & 7 \\
\hline
\end{tabular}

ceae is due to the highest number of species disseminated throughout the DRC (table V) (Bakwaye et al., 2013).

Similar to observations from other countries (Adekunle, 2008; Tabuti, 2008; Kodi et al., 2017), woody plants constituted the largest source of antimalarial medicinal plants. From an ecological perspective, the life-forms profile of the plant allows a better appreciation of ecological conditions in which they live (Kami Kanda et al., 2019). The high percentage of phanerophytes in antimalarial plants could be due to permanence of those plants throughout year (Mamadou et al., 2019). The predominance of woody species and phanerophytes shows a forest physiognomy (Kikufi et al., 2017; Lassa et al., 2019), while the therophytes correspond to the ruderal strategy (Kikufi and Lukoki, 2008; Masharabu et al., 2010). 
Although several plant parts were used in the Congo, leaves were the most commonly used in malaria treatment. This is similar to other studies from African countries that have demonstrated leaves to be the most frequently used plant part in plant remedies (Saotoing et al., 2011; Adia et al., 2014; Anywar et al., 2016). This contrasts with the findings of Ngarivhume et al. (2015) in Zimbabwe where roots were the most commonly used plant part. The preference for the utilization of leaves is justified by their effortless to collect, to store, and to process, but also, their action in photosynthesis and their bioactive compounds (Kayani et al., 2014; Bibi et al., 2015; Vijayakumar et al., 2015; Amjad et al., 2017; Faruque et al., 2019). Further, cutting leaves is less harmful to the plant development and growth (Alalwan et al., 2019).

It was found in this study that the most commonly used mode of preparation of antimalarial plants was decoction. This affirmation corroborates the reports from the other African countries (Koudouvo et al., 2011; Yetein et al., 2013; Alebie et al., 2017; Okello and Kang, 2019) and Asian countries (Bora et al., 2007; Ong et al., 2018). Decoction is largely used because of it is easy to prepare by mixing herbs with water, tea, or soup (Umair et al., 2019).

The primary administration route is oral. These results are consistent with the observations reported by other countries (Bora et al., 2007; Tor-anyiin et al., 2003; Idowu et al., 2010).
Among the recorded plant species, some have already been studied in vitro by Congolese Scientists, however, the phytochemical study of many antimalarial plant species has not yet been documented in vitro and/or in vivo.

\section{Conclusion}

This systematic review of medicinal plants provides a comprehensive insight into the existing antimalarial plants species in the Democratic Republic of the Congo (DRC). The 28 ethnobotanical studies published in the last two decades used in the study, highlighted the diversity of commonly used plant species with pharmacological effects, and their spatial distribution across the DRC (cultures and provinces), and represent an alternative mean for malaria prevention and a remedy for its treatment in the DRC. In addition, plants remain the major therapeutic remedy for malaria. Nevertheless, there are likely more sources of traditional knowledge and articles not published online that may contain precious information in the Phyto pharmacopeia against malaria that could serve as a basis for future studies.

\section{Acknowledgments}

The authors are grateful to all of the scholars whose work contributed to this systematic review, and Rusaati B. I. also thanks the Korea Forestry Promotion Institute (KOFPI) for providing his PhD scholarship.

\section{Appendix 1 - List of antimalarial plants}

\begin{tabular}{|c|c|c|c|c|c|c|c|c|}
\hline Plant family & Botanical name & $\begin{array}{l}\text { Morphological } \\
\text { type }\end{array}$ & $\begin{array}{l}\text { Life } \\
\text { form }\end{array}$ & $\begin{array}{l}\text { Part } \\
\text { used }\end{array}$ & $\begin{array}{l}\text { Preparation } \\
\text { mode }\end{array}$ & $\begin{array}{l}\text { Used } \\
\text { methods }\end{array}$ & Provinces & References \\
\hline Acanthaceae & $\begin{array}{l}\text { Hygrophila auriculata } \\
\text { (Schumach.) Heine }\end{array}$ & $\mathrm{H}$ & $\mathrm{Ch}$ & AP & NR & NR & 2 & $\begin{array}{l}\text { Karhagomba et al. (2013); } \\
\text { Manya et al. (2020) }\end{array}$ \\
\hline Acanthaceae & $\begin{array}{l}\text { Hypoestes triflora (Forssk.) } \\
\text { Roem. \& Schult. }\end{array}$ & $\mathrm{H}$ & $\mathrm{Ch}$ & WP & $\mathrm{De}$ & Oral & 2 & Manya et al. (2020) \\
\hline Acanthaceae & $\begin{array}{l}\text { Thomandersia hensii De Wild. } \\
\text { \& T. Durand }\end{array}$ & Sh & $\mathrm{Ph}$ & L & De & Oral & 4 & Ngbolua et al. (2014) \\
\hline Acanthaceae & Justicia insularis T. Anderson & $\mathrm{H}$ & Th & SB & $\mathrm{De}$ & Oral & 1 & Mbuyi et al. (2019) \\
\hline Amaranthaceae & Chenopodium album L. & $\mathrm{H}$ & Th & WP & $\mathrm{De}$ & Enema & 1 & Mbuyi et al. (2019) \\
\hline Amaranthaceae & $\begin{array}{l}\text { Chenopodium opulifolium } \\
\text { Schrad. ex W.D.J. Koch \& Ziz }\end{array}$ & $\mathrm{H}$ & Th & $\mathrm{L}$ & $\mathrm{De}$ & Oral & 2 & Manya et al. (2020) \\
\hline Amaranthaceae & Cyathula prostrata (L.) Blume & $\mathrm{H}$ & Th & L & $\mathrm{De}$ & Oral, noise & 6 & Konda et al. (2012) \\
\hline Amaranthaceae & $\begin{array}{l}\text { Dysphania ambrosioides (L.) } \\
\text { Mosyakin\& Clemants }\end{array}$ & $\mathrm{H}$ & Th & $\mathrm{L}$ & $\mathrm{De}$ & Oral & 2,3 & $\begin{array}{l}\text { Kasali et al. (2014a); Kasali et } \\
\text { al. (2014b); Manya et al. (2020) }\end{array}$ \\
\hline Amaryllidaceae & Allium sativum L. & $\mathrm{H}$ & $\mathrm{Ge}$ & Bulds & Pounding & NR & 2,3 & $\begin{array}{l}\text { Kasali et al. (2014a); } \\
\text { Kasali et al.(2014b) }\end{array}$ \\
\hline Amaryllidaceae & Allium cepa L. & $\mathrm{H}$ & $\mathrm{Ge}$ & WP & $\operatorname{lnf}$ & Enema & 1 & Mbuyi et al. (2019) \\
\hline Anacardiaceae & $\begin{array}{l}\text { Pseudospondias microcarpa } \\
\text { (A. Rich.) Engl.) }\end{array}$ & $\mathrm{T}$ & $\mathrm{Ph}$ & $L, B$ & $\mathrm{De}, \mathrm{Ma}$ & Oral, anal & 6 & Konda et al. (2012) \\
\hline Anacardiaceae & Mangifera indica L. & $\mathrm{T}$ & $\mathrm{Ph}$ & $L, R B, S B$ & $\mathrm{De}, \mathrm{Ma}$ & Oral & 1 & Mbuyi et al. (2019) \\
\hline Anisophylleaceae & $\begin{array}{l}\text { Anisophyllea pomifera Engl. } \\
\text { \& Brehmer }\end{array}$ & $\mathrm{T}$ & $\mathrm{Ph}$ & $\mathrm{L}, \mathrm{R}, \mathrm{SB}$ & $\mathrm{De}, \mathrm{Ma}$ & Oral, enema & 1 & $\begin{array}{l}\text { Chiribagula et al. (2017); } \\
\text { Kalonda et al. (2014); } \\
\text { Mbuyi et al. (2019) }\end{array}$ \\
\hline Annonaceae & $\begin{array}{l}\text { Monodora myristica (Gaertn.) } \\
\text { Dunal }\end{array}$ & $\mathrm{T}$ & $\mathrm{Ph}$ & $\mathrm{Fr}, \mathrm{Gr}$ & NR & NR & 4 & Ngbolua et al. (2015) \\
\hline Annonaceae & $\begin{array}{l}\text { Hexalobus monopetalus } \\
\text { (A. Rich.) Engl. \& Diels }\end{array}$ & Sh & $\mathrm{Ph}$ & $\mathrm{R}$ & De & Oral & 1 & $\begin{array}{l}\text { Mbuyi et al. (2019); } \\
\text { Kalonda et al. (2014) }\end{array}$ \\
\hline Annonaceae & Annona senegalensis Pers. & Sh & $\mathrm{Ph}$ & $\mathrm{R}, \mathrm{SB}$ & NR & Oral & 4 & $\begin{array}{l}\text { Ngbolua et al. (2015); } \\
\text { Ngbolua et al. (2016) }\end{array}$ \\
\hline Annonaceae & $\begin{array}{l}\text { Annickia chlorantha (Oliv.) } \\
\text { Setten \& Mass }\end{array}$ & $\mathrm{T}$ & $\mathrm{Ph}$ & SB & De & Oral & 4 & Ngbolua et al. (2014) \\
\hline Annonaceae & $\begin{array}{l}\text { Isolona hexaloba (Pierre) } \\
\text { Engl. \& Diels }\end{array}$ & $\mathrm{T}$ & $\mathrm{Ph}$ & SB & De & Oral & 4 & Ngbolua et al. (2014) \\
\hline Annonaceae & Monodora laurentii De Wild. & $\mathrm{T}$ & $\mathrm{Ph}$ & SB & De & Oral & 4 & Ngbolua et al. (2014) \\
\hline
\end{tabular}


Appendix 1 (continued)

\begin{tabular}{|c|c|c|c|c|c|c|c|c|}
\hline Plant family & Botanical name & $\begin{array}{l}\text { Morphological } \\
\text { type }\end{array}$ & $\begin{array}{l}\text { Life } \\
\text { form }\end{array}$ & $\begin{array}{l}\text { Part } \\
\text { used }\end{array}$ & $\begin{array}{l}\text { Preparation } \\
\text { mode }\end{array}$ & $\begin{array}{l}\text { Used } \\
\text { methods }\end{array}$ & Provinces & References \\
\hline Annonaceae & Uvaria scabrida Oliv. & $\mathrm{L}$ & $\mathrm{Ph}$ & SB & De & Oral & 4 & Ngbolua et al. (2014) \\
\hline Annonaceae & Xylopia aethiopica (Dunal) A. Rich. & $\mathrm{T}$ & $\mathrm{Ph}$ & SB & $\mathrm{De}$ & Oral & 1 & Mbuyi et al. (2019) \\
\hline Apiaceae & Centella asiatica (L.) Urb. & $\mathrm{H}$ & $\mathrm{Ch}$ & $L, R$ & $\mathrm{Ma}$ & NR & 1 & Kalonda et al. (2014) \\
\hline Apiaceae & Steganotaenia araliacea Hochst. & $\mathrm{T}$ & $\mathrm{Ph}$ & $L, R$ & De & Oral & 1 & Mbuyi et al. (2019) \\
\hline Apiaceae & $\begin{array}{l}\text { Agrocharis incognita (C. Norman) } \\
\text { Heywood and Jury) }\end{array}$ & $\mathrm{H}$ & Th & NR & NR & NR & 2 & Karhagomba et al. (2013) \\
\hline Apocynaceae & Alstonia boonei De Wild. & $\mathrm{T}$ & $\mathrm{Ph}$ & B & $\mathrm{De}$ & Oral & $4,7,10$ & $\begin{array}{l}\text { Terashima and Ichikawa (2003); } \\
\text { Ngbolua et al. (2014); } \\
\text { Mongeke et al. (2018) }\end{array}$ \\
\hline Apocynaceae & Mondia whitei (Hook. f.) Skeels & $\mathrm{L}$ & $\mathrm{Ph}$ & $\mathrm{B}$ & NR & NR & 4 & Ngbolua et al. (2019) \\
\hline Apocynaceae & Landolphia kirkii Dyer & $\mathrm{L}$ & $\mathrm{Ph}$ & $\mathrm{L}$ & De, Ma & Oral & 1 & Chiribagula et al. (2017) \\
\hline Apocynaceae & Landolphia parvifolia K. Schum. & $\mathrm{L}$ & $\mathrm{PH}$ & L & De & Oral & 1 & Mbuyi et al. (2019) \\
\hline Apocynaceae & Catharanthus roseus (L.) G. Don & Ssh & $\mathrm{Ph}$ & $L, R$ & De & Oral & $1,2,3,5$ & $\begin{array}{l}\text { Kasali et al. (2014a); Ngbolua } \\
\text { et al. (2013a); Kasali et al. } \\
\text { (2014b); Mbuyi et al. (2019) }\end{array}$ \\
\hline Apocynaceae & Rauvolfia caffra Sond & $\mathrm{T}$ & $\mathrm{Ph}$ & $L, R B, S B$ & $\mathrm{De}$ & Oral & 1 & Mbuyi et al. (2019) \\
\hline Apocynaceae & Rauvolfia vomitoria Afzel. & Sh & $\mathrm{Ph}$ & L, RB, SB & $\mathrm{De}$ & Oral & $1,3,4,6$ & $\begin{array}{l}\text { Kasali et al. (2014a); } \\
\text { Makumbelo et al. (2008); } \\
\text { Ilumbe Bayeli (2010); Kasika } \\
\text { et al. (2015); Mbuyi et al. (2019) }\end{array}$ \\
\hline Apocynaceae & $\begin{array}{l}\text { Landolphia congolensis (Stapf) } \\
\text { Pichon }\end{array}$ & $\mathrm{L}$ & $\mathrm{Ph}$ & $\mathrm{L}, \mathrm{S}$ & De & NR & 1 & Kalonda et al. (2014) \\
\hline Apocynaceae & $\begin{array}{l}\text { Diplorhynchus condylocarpon } \\
\text { (Müll. Arg.) Pichon }\end{array}$ & $\mathrm{T}$ & $\mathrm{Ph}$ & RB & De & Oral & 1 & Mbuyi et al. (2019) \\
\hline Apocynaceae & $\begin{array}{l}\text { Picralima nitida (Stapf) T. Durand } \\
\& \text { H. Durand }\end{array}$ & $\mathrm{T}$ & $\mathrm{Ph}$ & Se & Chewing & Oral & 4 & Ngbolua et al. (2014) \\
\hline Araceae & Amorphophallus angolensis N.E. Br. & $\mathrm{H}$ & $\mathrm{Ge}$ & Tubers & NR & Oral & 2 & Chifundera (2001) \\
\hline Arecaceae & Raphia sudanica A. Chev. & Sh & $\mathrm{Ph}$ & L & De & Oral & 5 & Nzuki (2016) \\
\hline Arecaceae & Raphia gentiliana De Wild. & $\mathrm{T}$ & $\mathrm{Ph}$ & NR & $\mathrm{De}$ & Oral & 5 & Nzuki (2016) \\
\hline Aristolochiaceae & Aristolochia hockii De Wild. & $\mathrm{H}$ & $\mathrm{Ch}$ & RB & $\mathrm{De}$ & Oral & 1 & Mbuyi et al. (2019) \\
\hline Aristolochiaceae & Aristolochia sp. & $\mathrm{H}$ & $\mathrm{Ph}$ & Se & $\mathrm{De}$ & NR & 3 & Kasali et al. (2014a) \\
\hline Asphodelaceae & Aloe sp. & $\mathrm{H}$ & $\mathrm{Ge}$ & AP & $\mathrm{De}$ & NR & 2 & Kasali et al. (2014b) \\
\hline Asphodelaceae & Aloe buettneri A. Berger & $\mathrm{H}$ & $\mathrm{Ge}$ & $\mathrm{L}$ & sap & Friction & 5 & Nzuki (2016) \\
\hline Asphodelaceae & Aloe dawei A. Berger & $\mathrm{H}$ & $\mathrm{Ge}$ & $\mathrm{L}$ & $\mathrm{De}$ & NR & 3 & Kasali et al. (2014a) \\
\hline Asphodelaceae & Aloe christianii Reynolds & $\mathrm{H}$ & $\mathrm{Ge}$ & $L, R$ & $\mathrm{De}$ & Oral & 1 & Mbuyi et al. (2019) \\
\hline Asteraceae & $\begin{array}{l}\text { Conyza sumatrensis (S.F. Blake) } \\
\text { Pruski \& G. Sancho }\end{array}$ & $\mathrm{H}$ & $\mathrm{Ch}$ & L & De & Oral & 3 & $\begin{array}{l}\text { Kasali et al. (2014a); } \\
\text { Kasika et al. (2015) }\end{array}$ \\
\hline Asteraceae & $\begin{array}{l}\text { Crassocephalum monthuosum } \\
\text { (S. Moore) Milme-Redh }\end{array}$ & $\mathrm{H}$ & $\mathrm{Ch}$ & $\mathrm{L}$ & De, Ma, Ash & Oral & 2,3 & $\begin{array}{l}\text { Kasali et al. (2014a); } \\
\text { Manya et al. (2020) }\end{array}$ \\
\hline Asteraceae & Mikania cordata (Burm. f.) B.L. Rob. & L & $\mathrm{Ch}$ & $\mathrm{L}$ & De & NR & 3 & Kasali et al. (2014a) \\
\hline Asteraceae & $\begin{array}{l}\text { Tithonia diversifolia (Hemsl.) } \\
\text { A. Cray. }\end{array}$ & Ssh & $\mathrm{Ch}$ & $L, R$ & $\mathrm{De}, \mathrm{Ma}$ & Oral, enema & $1,2,3$ & $\begin{array}{l}\text { Kasali et al. (2014a); Kasali et al. } \\
\text { (2014b); Mbuyi et al. (2019) }\end{array}$ \\
\hline Asteraceae & $\begin{array}{l}\text { Dichrocephala integrifolia (L. f.) } \\
\text { O. Ktze. }\end{array}$ & $\mathrm{H}$ & $\mathrm{HC}$ & NR & NR & Hit a patient & 3,7 & $\begin{array}{l}\text { Kasika et al. (2015); } \\
\text { Terashima and Ichikawa (2003) }\end{array}$ \\
\hline Asteraceae & Chamaemelum nobile (L.) All. & $\mathrm{H}$ & $\mathrm{Ph}$ & $\mathrm{Fl}$ & Inf & NR & 2 & Kasali et al. (2014b) \\
\hline Asteraceae & Achillea millefolium L. & $\mathrm{H}$ & $\mathrm{Ph}$ & $\mathrm{L}$ & $\mathrm{De}$ & NR & 3 & Kasali et al. (2014a) \\
\hline Asteraceae & Artemisia annua L. & $\mathrm{H}$ & $\mathrm{Ph}$ & $\mathrm{L}$ & $\operatorname{lnf}$ & Oral & 2,3 & $\begin{array}{l}\text { Kasali et al. (2014a); } \\
\text { Karhagomba et al. (2013); } \\
\text { Kasali et al. (2014b); } \\
\text { Manya et al. (2020) }\end{array}$ \\
\hline Asteraceae & $\begin{array}{l}\text { Baccharoides adoensis } \\
\text { (Sch. Bip. ex Walp.) H. Rob. }\end{array}$ & $\mathrm{H}$ & $\mathrm{Ph}$ & $\mathrm{L}$ & $\mathrm{Ma}$ & Enema, oral & 1 & $\begin{array}{l}\text { Mbuyi et al. (2019); } \\
\text { Muya et al. (2014) }\end{array}$ \\
\hline Asteraceae & Microglossa pyrifolia (Lam.) Kuntze & $\mathrm{T}$ & $\mathrm{Ph}$ & $\mathrm{L}$ & De & NR & 3 & Kasali et al. (2014a) \\
\hline Asteraceae & Sambucus canadensis L. & $\mathrm{T}$ & $\mathrm{Ph}$ & L & De & NR & 3 & Kasali et al. (2014a) \\
\hline Asteraceae & Mikania microptera DC. & $\mathrm{H}$ & $\mathrm{Ph}$ & $\mathrm{L}$ & sap & Put in eyes & 7 & Terashima and Ichikawa (2003) \\
\hline Asteraceae & Matricaria chamomilla L. & $\mathrm{H}$ & $\mathrm{Ph}$ & L, Fr & De, $\ln f$ & NR & 2,3 & $\begin{array}{l}\text { Kasali et al. (2014a); } \\
\text { Kasali et al. (2014b) }\end{array}$ \\
\hline Asteraceae & Vernonia amygdalina Del. & Sh & $\mathrm{Ph}$ & $L, R$ & De, $\ln f$ & Oral & $1,2,3,5$ & $\begin{array}{l}\text { Kasali et al. (2014a); } \\
\text { Karhagomba et al. (2013); } \\
\text { Ngbolua et al. (2013b); Kasali } \\
\text { et al. (2014b); Ngbolua et al. } \\
\text { (2014); Manya et al. (2020); } \\
\text { Kalonda et al. (2014) }\end{array}$ \\
\hline Asteraceae & $\begin{array}{l}\text { Acanthospermum glabratum (DC.) } \\
\text { Wild }\end{array}$ & $\mathrm{H}$ & Th & $\mathrm{L}$ & $\mathrm{De}$ & Oral & 2 & Manya et al. (2020) \\
\hline Asteraceae & Ageratum conyzoides (L.) L. & $\mathrm{H}$ & Th & $\mathrm{L}$ & $\mathrm{De}$ & NR & 2,3 & $\begin{array}{l}\text { Kasali et al. (2014a); } \\
\text { Kasali et al. (2014b) }\end{array}$ \\
\hline
\end{tabular}


Bois et Forêts des Tropiques - ISSN: L-0006-579X

Volume $347-1^{\text {st }}$ quarter - February 2021 - p. 11-27

\section{Appendix 1 (continued)}

\begin{tabular}{|c|c|c|c|c|c|c|c|c|}
\hline Plant family & Botanical name & $\begin{array}{l}\text { Morphological } \\
\text { type }\end{array}$ & $\begin{array}{l}\text { Life } \\
\text { form }\end{array}$ & $\begin{array}{l}\text { Part } \\
\text { used }\end{array}$ & $\begin{array}{l}\text { Preparation } \\
\text { mode }\end{array}$ & $\begin{array}{l}\text { Used } \\
\text { methods }\end{array}$ & Provinces & References \\
\hline Asteraceae & Bidens pilosa $\mathrm{L}$. & $\mathrm{H}$ & Th & L & De & Oral & $1,2,3$ & $\begin{array}{l}\text { Kasali et al. (2014a); Kasika } \\
\text { et al. (2015); Kasali et al. } \\
\text { (2014b); Mbuyi et al. (2019); } \\
\text { Manya et al. (2020); } \\
\text { Kalonda et al. (2014) }\end{array}$ \\
\hline Asteraceae & Cyanthillium cinereum (L.) H. Rob. & $\mathrm{H}$ & Th & L & Ma & Oral & 1 & Mbuyi et al. (2019) \\
\hline Asteraceae & Synedrella nodiflora (L.) Gaertn. & $\mathrm{H}$ & Th & $\mathrm{L}$ & De & Oral & 2 & Manya et al. (2020) \\
\hline Asteraceae & Artemisia sp. & $\mathrm{H}$ & Th & WP & De & NR & 5 & Ngbolua et al. (2013a) \\
\hline Asteraceae & $\begin{array}{l}\text { Aspilia kotschyi (Sch. Bip. } \\
\text { ex Hochst.) Oliv. }\end{array}$ & $\mathrm{H}$ & Th & WP & De & Oral & 2 & Manya et al. (2020) \\
\hline Asteraceae & Bidens oligoflora (Klatt) Wild & $\mathrm{H}$ & Th & WP & De & Oral & 2 & Manya et al. (2020) \\
\hline Asteraceae & $\begin{array}{l}\text { Crassocephalum picridifolium } \\
\text { (DC.) S. Moore }\end{array}$ & $\mathrm{H}$ & Th & WP & De & Oral & 2 & Manya et al. (2020) \\
\hline Asteraceae & Polydora serratuloides (DC.) H. Rob & $\mathrm{H}$ & Th & WP & De & Oral & 2 & Manya et al. (2020) \\
\hline Asteraceae & $\begin{array}{l}\text { Porphyrostemma chevalieri } \\
\text { (0. Hoffm.) Hutch. \& Dalziel }\end{array}$ & $\mathrm{H}$ & & $\mathrm{L}$ & De & Oral & 2 & Manya et al. (2020) \\
\hline Asteraceae & $\begin{array}{l}\text { Anisopappus chinensis } \\
\text { Hook \& Arn. }\end{array}$ & $\mathrm{H}$ & & WP & NR & NR & 11 & Lusakibanza (2012) \\
\hline Bignoniaceae & Spathodea campanulata P. Beauv. & $T$ & $\mathrm{Ph}$ & L & De, Inf & Oral & 2 & Manya et al. (2020) \\
\hline Bignoniaceae & Newbouldia laevis (P. Beauv.) Seem. & T & $\mathrm{Ph}$ & $\mathrm{R}$ & Ma & NR & 4 & Makumbelo et al. (2008) \\
\hline Boraginaceae & Cynoglossum lanceolatum Forssk. & $\mathrm{H}$ & Th & L & De & NR & 3 & Kasali et al. (2014a) \\
\hline Bromeliaceae & Ananas cosmesus (L.) Merr. & $\mathrm{H}$ & $\mathrm{Ch}$ & $\mathrm{L}, \mathrm{Fr}$ & Exp, ext & NR & 2,3 & $\begin{array}{l}\text { Kasali et al. (2014a); } \\
\text { Kasali et al. (2014b) }\end{array}$ \\
\hline Caricaceae & Carica papaya L. & $\mathrm{T}$ & $\mathrm{Ph}$ & $\mathrm{L}, \mathrm{R}, \mathrm{Fl}$ & De, Inf, Ma & Oral & $1,2,3$ & $\begin{array}{l}\text { Kasali et al. (2014a); Kasika et al. } \\
\text { (2015); Kasali et al. (2014b); } \\
\text { Mbuyi et al. (2019); Manya et al. } \\
\text { (2020); Ilumbe Bayeli (2010); } \\
\text { Muya et al. (2014); } \\
\text { Kalonda et al. (2014) }\end{array}$ \\
\hline Chrysobalanaceae & $\begin{array}{l}\text { Parinari curatellifolia Planch. } \\
\text { ex Benth. }\end{array}$ & T & $\mathrm{Ph}$ & $R$ & De & Oral & 1 & Mbuyi et al. (2019) \\
\hline Clusiaceae & Garcinia huillensis Welw. & $T$ & $\mathrm{Ph}$ & L, RB & De & Oral & 2 & Manya et al. (2020) \\
\hline Clusiaceae & Lebrunia buchaie Staner & $T$ & $\mathrm{Ph}$ & NR & NR & NR & 2 & Mangambu et al. (2015b) \\
\hline Clusiaceae & Garcinia kola Heckel & $\mathrm{T}$ & $\mathrm{Ph}$ & $\mathrm{SB}, \mathrm{Fr}$ & De & Chewing, oral & 4,6 & $\begin{array}{l}\text { Ngbolua et al. (2015); Ngbolua } \\
\text { et al. (2016); Ngbolua et al. } \\
\text { (2019); Ilumbe Bayeli (2010) }\end{array}$ \\
\hline Combretaceae & $\begin{array}{l}\text { Combretum haullevilleanum } \\
\text { De Wild. }\end{array}$ & Sh & $\mathrm{Ph}$ & L, S, SB & De & Oral & 1 & Mbuyi et al. (2019) \\
\hline Combretaceae & Combretum zeyheri Sond. & $T$ & $\mathrm{Ph}$ & L, S, SB & De & Oral & 1 & Mbuyi et al. (2019) \\
\hline Combretaceae & Combretum molle R. Br. ex G. Don & $\mathrm{T}$ & $\mathrm{Ph}$ & L, SB & De & Oral & 2 & Manya et al. (2020) \\
\hline Combretaceae & Terminalia mollis M.A. Lawson & T & $\mathrm{Ph}$ & L, SB & De & Oral & 1 & Mbuyi et al. (2019) \\
\hline Commelinaceae & Tradescantia zebrina Bosse & $\mathrm{H}$ & $\mathrm{Ch}$ & L, Fr & De & NR & 3 & Kasali et al. (2014a) \\
\hline Convolvulaceae & Ipomoea indica (Burm.) Merr. & $\mathrm{H}$ & $\mathrm{Ch}$ & WP & De & Oral & 2 & Manya et al. (2020) \\
\hline Crassulaceae & Kalanchoe crenata (Andrews) Haw. & $\mathrm{H}$ & $\mathrm{Ch}$ & $\mathrm{L}$ & De, Sap & $\begin{array}{l}\text { Oral, } \\
\text { instillation }\end{array}$ & 2 & Manya et al. (2020) \\
\hline Cucurbitaceae & Oreosyce africana Hook. f. & L & $\mathrm{Ph}$ & WP & $\mathrm{De}$ & Oral & 2 & Chifundera (2001) \\
\hline Cucurbitaceae & Momordica foetida Schumach. & $\mathrm{H}$ & Th & $\mathrm{L}$ & ext & NR & 2 & Kasali et al. (2014b) \\
\hline Cupressaceae & Cupressus lusitanica Mill. & T & $\mathrm{Ph}$ & L & De, Inf & NR & 2,3 & $\begin{array}{l}\text { Kasali et al. (2014a); } \\
\text { Kasali et al. (2014b) }\end{array}$ \\
\hline Ebenaceae & $\begin{array}{l}\text { Diospyros mespiliformis Hochst. } \\
\text { ex A. DC. }\end{array}$ & $\mathrm{T}$ & $\mathrm{Ph}$ & $\mathrm{R}$ & $\mathrm{Ma}$ & Oral & 1 & Mbuyi et al. (2019) \\
\hline Euphorbiaceae & Acalypha brachiata Krauss & Sh & $\mathrm{Hc}$ & L & De & Oral & 1 & Mbayo et al. (2016) \\
\hline Euphorbiaceae & Croton sp. & $T$ & $\mathrm{Ph}$ & B & De & NR & 3 & Kasali et al. (2014a) \\
\hline Euphorbiaceae & Acalypha homblei De Wild. & $\mathrm{H}$ & $\mathrm{Ph}$ & L & De & Oral & 2 & Manya et al. (2020) \\
\hline Euphorbiaceae & $\begin{array}{l}\text { Alchornea cordifolia (Schumach. } \\
\text { \& Thonn.) Müll. Arg. }\end{array}$ & Sh & $\mathrm{Ph}$ & L & De & Oral & 6 & Konda et al. (2012) \\
\hline Euphorbiaceae & Ricinus communis L. & Sh & $\mathrm{Ph}$ & $\mathrm{L}$ & $\mathrm{Ma}$ & Bath & 1 & Mbuyi et al. (2019) \\
\hline Euphorbiaceae & $\begin{array}{l}\text { Antidesma venosum E. Mey. } \\
\text { ex Tul. }\end{array}$ & T & $\mathrm{Ph}$ & $L, R, R B$ & De, Inf & Oral & 1,2 & $\begin{array}{l}\text { Mbuyi et al. (2019); Mbayo } \\
\text { et al. (2016); Manya et al. } \\
\text { (2019); Kalonda et al. (2014) }\end{array}$ \\
\hline Euphorbiaceae & Maprounea africana Müll. Arg. & $T$ & $\mathrm{Ph}$ & L, RB & De & Oral & 2 & Manya et al. (2020) \\
\hline Euphorbiaceae & Jatropha curcas L. & Sh & $\mathrm{Ph}$ & L, SB & De & $\begin{array}{l}\text { Rub the leaves } \\
\text { on the body, } \\
\text { oral }\end{array}$ & $1,5,8$ & $\begin{array}{l}\text { Ndombe et al. (2016); Mbuyi } \\
\text { et al. (2019); Mbayo et al. } \\
\text { (2016); Nzuki (2016) }\end{array}$ \\
\hline Euphorbiaceae & $\begin{array}{l}\text { Phyllanthus muellerianus } \\
\text { (Kuntze) Exell. }\end{array}$ & Sh & $\mathrm{Ph}$ & L, SB & De, Ma, Gri & $\begin{array}{l}\text { Oral, } \\
\text { fomentation }\end{array}$ & 1 & $\begin{array}{l}\text { Chiribagula et al. (2017); } \\
\text { Mbuyi et al. (2019); } \\
\text { Mbayo et al. (2016) }\end{array}$ \\
\hline Euphorbiaceae & Manihot esculenta $\mathrm{L}$. & Sh & $\mathrm{Ph}$ & NR & NR & NR & 5 & Nzuki (2016) \\
\hline Euphorbiaceae & Euphorbia ingens E. Mey. ex Boiss. & $T$ & $\mathrm{Ph}$ & $\mathrm{R}$ & $\mathrm{Ma}$ & Oral & 1 & Mbuyi et al. (2019) \\
\hline
\end{tabular}


Appendix 1 (continued)

\begin{tabular}{|c|c|c|c|c|c|c|c|c|}
\hline Plant family & Botanical name & $\begin{array}{l}\text { Morphological } \\
\text { type }\end{array}$ & $\begin{array}{l}\text { Life } \\
\text { form }\end{array}$ & $\begin{array}{l}\text { Part } \\
\text { used }\end{array}$ & $\begin{array}{l}\text { Preparation } \\
\text { mode }\end{array}$ & $\begin{array}{l}\text { Used } \\
\text { methods }\end{array}$ & Provinces & References \\
\hline Euphorbiaceae & Euphorbia tirucalli L. & $\mathrm{T}$ & $\mathrm{Ph}$ & $\mathrm{R}$ & $\mathrm{Ma}$ & Oral & 1 & $\begin{array}{l}\text { Mbuyi et al. (2019); } \\
\text { Muya et al. (2014) }\end{array}$ \\
\hline Euphorbiaceae & Euphorbia hirta L. & $\mathrm{H}$ & Th & $\mathrm{R}$ & $\mathrm{Ma}$ & Oral & 1 & Mbuyi et al. (2019) \\
\hline Euphorbiaceae & Euphorbia parviflora L. & $\mathrm{H}$ & & AP & Ma (oil) & NR & 2 & Kasali et al. (2014b) \\
\hline Fabaceae & $\begin{array}{l}\text { Indigofera arrecta Hochst. } \\
\text { ex A. Rich. }\end{array}$ & $\mathrm{H}$ & $\mathrm{Ch}$ & L & $\operatorname{lnf}$ & NR & 2 & Kasali et al. (2014b) \\
\hline Fabaceae & $\begin{array}{l}\text { Hylodesmum repandum (Vahl) } \\
\text { H. Ohashi \& R.R. Mill }\end{array}$ & Ssh & $\mathrm{Ch}$ & $\mathrm{L}, \mathrm{FL}$ & De, Inf & Oral & 2 & Manya et al. (2020) \\
\hline Fabaceae & Dialium angolense Oliv. & $\mathrm{T}$ & $\mathrm{Ph}$ & L & De & Oral & 2 & Manya et al. (2020) \\
\hline Fabaceae & $\begin{array}{l}\text { Julbernardia paniculata (Benth.) } \\
\text { Troupin }\end{array}$ & $\mathrm{T}$ & $\mathrm{Ph}$ & $\mathrm{L}$ & De, Inf & Oral & 2 & Manya et al. (2020) \\
\hline Fabaceae & Parkia bicolor A. Chev. & $\mathrm{T}$ & $\mathrm{Ph}$ & $\mathrm{L}$ & De & Oral & 1 & Mbuyi et al. (2019) \\
\hline Fabaceae & $\begin{array}{l}\text { Senna spectabilis (DC.) H.S. } \\
\text { Irwin \& Barneby }\end{array}$ & $\mathrm{H}$ & $\mathrm{Ph}$ & $\mathrm{L}$ & De & NR & 2 & Kasali et al. (2014b) \\
\hline Fabaceae & $\begin{array}{l}\text { Bobgunnia madagascariensis } \\
\text { (Desv.) J.H. Kirkbr. and Wiersema }\end{array}$ & $\mathrm{T}$ & $\mathrm{Ph}$ & $L, R$ & De, Ma, Spray & Oral, bath & 1 & $\begin{array}{l}\text { Chiribagula et al. (2017); } \\
\text { Mbuyi et al. (2019) }\end{array}$ \\
\hline Fabaceae & Dalbergia nitidula Baker & Sh & $\mathrm{Ph}$ & L, R & De & NR & 1 & Kalonda et al. (2014) \\
\hline Fabaceae & Bauhinia reticulata DC. & $\mathrm{T}$ & $\mathrm{Ph}$ & $L, R, S B$ & De, Ma & Oral & 1,4 & $\begin{array}{l}\text { Mbuyi et al. (2019); Ngbolua et } \\
\text { al. (2014); Muya et al. (2014) }\end{array}$ \\
\hline Fabaceae & Pterocarpus angolensis DC. & $\mathrm{T}$ & $\mathrm{Ph}$ & $L, R, S B$ & $\mathrm{De}$ & Oral & 1 & Chiribagula et al. (2017) \\
\hline Fabaceae & Pterocarpus tinctorius Welw. & $\mathrm{T}$ & $\mathrm{Ph}$ & $L, R, S B$ & De & Oral & 1 & Mbuyi et al. (2019) \\
\hline Fabaceae & Senna occidentalis (L.) Link & Ssh & $\mathrm{Ph}$ & $L, R, S B$ & De, Ma & Oral & $\begin{array}{l}1,2,3 \\
5,6\end{array}$ & $\begin{array}{l}\text { Manya et al. (2020); Nzuki } \\
\text { (2016); Konda et al. (2012); } \\
\text { Kasali et al. (2014a); Kasali et } \\
\text { al. (2014b); Chiribagula et al. } \\
\text { (2017); Mbuyi et al. (2019) }\end{array}$ \\
\hline Fabaceae & Cajanus cajan (L.) Mill sp. & Sh & $\mathrm{Ph}$ & $\begin{array}{l}\mathrm{L}, \mathrm{R}, \mathrm{SB}, \\
\mathrm{Gr}\end{array}$ & $\begin{array}{l}\text { Milling and } \\
\text { maceration }\end{array}$ & $\begin{array}{l}\text { Oral, } \\
\text { instillation }\end{array}$ & 1 & $\begin{array}{l}\text { Chiribagula et al. (2017); Mbuyi } \\
\text { et al. (2019); Muya et al. (2014) }\end{array}$ \\
\hline Fabaceae & $\begin{array}{l}\text { Isoberlinia angolensis (Welw. } \\
\text { ex Benth.) Hoyle \& Brenan }\end{array}$ & $\mathrm{T}$ & $\mathrm{Ph}$ & $\mathrm{L}, \mathrm{RB}$ & De & Oral & 2 & Manya et al. (2020) \\
\hline Fabaceae & Acacia polyacantha Willd. & T & $\mathrm{Ph}$ & L, RB, SB & De, Inf, Ma & Oral & 1 & $\begin{array}{l}\text { Chiribagula et al. (2017); Mbuyi } \\
\text { et al. (2019); Muya et al. (2014) }\end{array}$ \\
\hline Fabaceae & Cassia sieberiana L. & $\mathrm{T}$ & $\mathrm{Ph}$ & L, SB & De & Oral & 1 & Mbuyi et al. (2019) \\
\hline Fabaceae & Dalbergia boehmii Taub. & T & $\mathrm{Ph}$ & L, SB & De, Ma & Oral & 1 & $\begin{array}{l}\text { Mbuyi et al. (2019); Kalonda et } \\
\text { al. (2014); Manya et al. (2020) }\end{array}$ \\
\hline Fabaceae & Acacia buchananii Harms & $\mathrm{T}$ & $\mathrm{Ph}$ & $\mathrm{R}$ & $\mathrm{De}, \mathrm{Ma}$ & Enema & 1 & Mbuyi et al. (2019) \\
\hline Fabaceae & Acacia karroo Hayne & Sh & $\mathrm{Ph}$ & $\mathrm{R}$ & $\mathrm{De}, \mathrm{Ma}$ & Enema & 1 & Mbuyi et al. (2019) \\
\hline Fabaceae & $\begin{array}{l}\text { Amblygonocarpus andongensis } \\
\text { (Oliv.) Exell \& Torre }\end{array}$ & $\mathrm{T}$ & $\mathrm{Ph}$ & $\mathrm{R}$ & De & Oral & 1 & Mbuyi et al. (2019) \\
\hline Fabaceae & Entada abyssinica A. Rich. & Sh & $\mathrm{Ph}$ & $\mathrm{R}$ & Spray & $\begin{array}{l}\text { Instillation } \\
\text { (ear, nose) }\end{array}$ & 1 & $\begin{array}{l}\text { Chiribagula et al. (2017); } \\
\text { Mbuyi et al. (2019) }\end{array}$ \\
\hline Fabaceae & $\begin{array}{l}\text { Pericopsis angolensis (Baker) } \\
\text { Meeuwen }\end{array}$ & T & $\mathrm{Ph}$ & $\mathrm{R}$ & De, Ma & Oral & 1 & Mbuyi et al. (2019) \\
\hline Fabaceae & Senna alata (L.) Roxb. & $\mathrm{T}$ & $\mathrm{Ph}$ & $\mathrm{R}$ & De & Oral & 1 & Mbuyi et al. (2019) \\
\hline Fabaceae & Erythrina abyssinica DC. & $\mathrm{T}$ & $\mathrm{Ph}$ & $\mathrm{R}, \mathrm{B}, \mathrm{Fr}$ & De, Inf, Ma & Oral, enema & $1,2,3$ & $\begin{array}{l}\text { Kasali et al. (2014a); Kasali et } \\
\text { al. (2014b); Mbuyi et al. (2019) }\end{array}$ \\
\hline Fabaceae & $\begin{array}{l}\text { Albizia adianthifolia (Schum.) } \\
\text { W.F. Wight }\end{array}$ & $\mathrm{T}$ & $\mathrm{Ph}$ & $\mathrm{RB}$ & De & $\begin{array}{l}\text { Oral, } \\
\text { fumigation, } \\
\text { bath }\end{array}$ & 1 & $\begin{array}{l}\text { Chiribagula et al. (2017); } \\
\text { Mbuyi et al. (2019) }\end{array}$ \\
\hline Fabaceae & Pentaclethra macrophylla Benth. & $\mathrm{T}$ & $\mathrm{Ph}$ & RB & De & Oral & 4 & Ngbolua et al. (2014) \\
\hline Fabaceae & Bauhinia thonningii Schum. & $\mathrm{T}$ & $\mathrm{Ph}$ & $\mathrm{RB}, \mathrm{SB}$ & De, Ma & Oral & 1 & $\begin{array}{l}\text { Mbuyi et al. (2019); } \\
\text { Muya et al. (2014) }\end{array}$ \\
\hline Fabaceae & Dalbergia chapelieri Baill. & Sh & $\mathrm{Ph}$ & S & $\operatorname{lnf}$ & Oral & 2 & Manya et al. (2020) \\
\hline Fabaceae & Arachis hypogaea L. & $\mathrm{H}$ & Th & Se & Paste & NR & $2,3,5$ & $\begin{array}{l}\text { Kasali et al. (2014a); Kasali } \\
\text { et al. (2014b); Nzuki (2016) }\end{array}$ \\
\hline Fabaceae & Droogmansia giorgii De Wild. & Ssh & & $L, R, S B$ & De & Oral & 1 & Mbuyi et al. (2019) \\
\hline Fabaceae & Baphia capparidifolia Baker & $\mathrm{L}$ & & $\mathrm{L}, \mathrm{SB}$ & De & Oral & 1 & Mbuyi et al. (2019) \\
\hline Fabaceae & $\begin{array}{l}\text { Rhynchosia insignis (0. Hoffm.) } \\
\text { R.E. Fr. }\end{array}$ & $\mathrm{H}$ & & $\mathrm{R}$ & $\mathrm{Ma}$ & Oral & 1 & Muya et al. (2014) \\
\hline Hypericaceae & Psorospermum corymbiferum Hochr. & Sh & $\mathrm{Ph}$ & $\mathrm{L}$ & $\operatorname{lnf}$ & Oral & 2 & Manya et al. (2020) \\
\hline Hypericaceae & $\begin{array}{l}\text { Harungana madagascariensis } \\
\text { Lam. ex Poir. }\end{array}$ & $\mathrm{T}$ & $\mathrm{Ph}$ & $L, R, S B$ & De & Oral & $1,2,6$ & $\begin{array}{l}\text { Kasali et al. (2014b); Muya et al. } \\
\text { (2014); Konda et al. (2012); } \\
\text { Mbuyi et al. (2019) }\end{array}$ \\
\hline Icacinaceae & Pyrenacantha staudtii (Engl.) Engl. & $\mathrm{L}$ & $\mathrm{Ph}$ & $\mathrm{L}$ & $\mathrm{De}$ & Oral & 6 & Ilumbe Bayeli (2010) \\
\hline Lamiaceae & $\begin{array}{l}\text { Kalaharia uncinata (Schinz) } \\
\text { Moldenke }\end{array}$ & Sh & Ch & $\mathrm{L}$ & De & Oral & 1 & Mbuyi et al. (2019) \\
\hline Lamiaceae & Ocimum gratissimum L. & Ssh & $\mathrm{Ch}$ & $\mathrm{L}$ & $\begin{array}{l}\text { De, Inf, Ma, } \\
\text { Gri }\end{array}$ & $\begin{array}{l}\text { Poultice, oral, } \\
\text { enema, bath }\end{array}$ & 1 & $\begin{array}{l}\text { Chiribagula et al. (2017); } \\
\text { Manya et al. (2020) }\end{array}$ \\
\hline Lamiaceae & Tetradenia riparia (Hochst.) Codd & $\mathrm{T}$ & $\mathrm{Ph}$ & $\mathrm{L}$ & $\operatorname{Exp}$ & NR & 2,3 & $\begin{array}{l}\text { Kasali et al. (2014a); } \\
\text { Kasali et al. (2014b) }\end{array}$ \\
\hline
\end{tabular}


Bois et Forêts des Tropiques - ISSN: L-0006-579X

Volume $347-1^{\text {st }}$ quarter - February 2021 - p. 11-27

\section{Appendix 1 (continued)}

\begin{tabular}{|c|c|c|c|c|c|c|c|c|}
\hline Plant family & Botanical name & $\begin{array}{l}\text { Morphological } \\
\text { type }\end{array}$ & $\begin{array}{l}\text { Life } \\
\text { form }\end{array}$ & $\begin{array}{l}\text { Part } \\
\text { used }\end{array}$ & $\begin{array}{l}\text { Preparation } \\
\text { mode }\end{array}$ & $\begin{array}{l}\text { Used } \\
\text { methods }\end{array}$ & Provinces & References \\
\hline Lamiaceae & Vitex madiensis Oliv. & Sh & $\mathrm{Ph}$ & $\mathrm{L}$ & De & $\begin{array}{l}\text { Oral, spray } \\
\text { on the body }\end{array}$ & 1 & $\begin{array}{l}\text { Mbuyi et al. (2019); } \\
\text { Kalonda et al. (2014) }\end{array}$ \\
\hline Lamiaceae & Mentha piperita L. & $\mathrm{H}$ & Th & L & $\mathrm{De}$ & NR & 2,3 & $\begin{array}{l}\text { Kasali et al. (2014a); } \\
\text { Kasali et al. (2014b) }\end{array}$ \\
\hline Lamiaceae & Ocimum americanum L. & $\mathrm{H}$ & Th & L, RB & Gri & $\begin{array}{l}\text { Rub the } \\
\text { leaves all } \\
\text { over the body }\end{array}$ & 1 & Mbuyi et al. (2019) \\
\hline Lamiaceae & Leucas martinicensis (Jacq.) R. Br. & $\mathrm{H}$ & Th & L, S & $\mathrm{De}$ & NR & 3 & Kasali et al. (2014a) \\
\hline Lamiaceae & $\begin{array}{l}\text { Plectranthus barbatus var. grandis } \\
\text { (L.H. Cramer) Lukhoba \& A.J. Paton }\end{array}$ & $\mathrm{H}$ & & $\mathrm{L}, \mathrm{R}$ & $\mathrm{De}$ & $\begin{array}{l}\text { Oral, } \\
\text { suppository }\end{array}$ & 1 & $\begin{array}{l}\text { Mbuyi et al. (2019); } \\
\text { Muya et al. (2014) }\end{array}$ \\
\hline Lauraceae & Persea americana Mill. & $\mathrm{T}$ & $\mathrm{Ph}$ & $\mathrm{L}, \mathrm{Fr}$ & $\mathrm{De}$ & Oral & $2,3,6$ & $\begin{array}{l}\text { Kasali et al. (2014a); Kasali et } \\
\text { al.(2014b); Ilumbe Bayeli (2010) }\end{array}$ \\
\hline Loganiaceae & Strychnos cocculoides Baker & Sh & $\mathrm{Ph}$ & RB & $\mathrm{De}$ & Oral & 1 & $\begin{array}{l}\text { Mbuyi et al. (2019); } \\
\text { Kalonda et al. (2014) }\end{array}$ \\
\hline Loganiaceae & Strychnos icaja Baill. & $\mathrm{L}$ & $\mathrm{Ph}$ & RB & NR & NR & 6 & Lusakibanza Manzo (2012) \\
\hline Loganiaceae & Strychnos potatorum L. f. & $\mathrm{T}$ & $\mathrm{Ph}$ & RB & De, powder & $\begin{array}{l}\text { Oral, } \\
\text { instillation } \\
\text { (eyes, noise) }\end{array}$ & 1 & Mbuyi et al. (2019) \\
\hline Loganiaceae & Strychnos spinosa Lam. & $\mathrm{T}$ & $\mathrm{Ph}$ & RB & De, powder & $\begin{array}{l}\text { Oral, } \\
\text { instillation } \\
\text { (eyes, noise) }\end{array}$ & 1 & Mbuyi et al. (2019) \\
\hline Lycopodiaceae & Lycopodium clavatum L. & $\mathrm{H}$ & & NR & NR & NR & 2 & Mangambu et al. (2012) \\
\hline Malvaceae & Sida acuta Burm. f. & Ssh & $\mathrm{Ch}$ & NR & NR & NR & 3 & Kasika et al. (2015) \\
\hline Melastomataceae & Memecylon flavovirens Baker & Sh & $\mathrm{Ph}$ & $\mathrm{L}, \mathrm{R}$ & Ma & Oral & 1 & Mbuyi et al. (2019) \\
\hline Meliaceae & $\begin{array}{l}\text { Ekebergia benguelensis Welw. } \\
\text { ex C. DC. }\end{array}$ & $\mathrm{T}$ & $\mathrm{Ph}$ & $\mathrm{L}$ & De & NR & 1 & Kalonda et al. (2014) \\
\hline Meliaceae & Melia azedarach L. & $\mathrm{T}$ & $\mathrm{Ph}$ & $\mathrm{L}$ & NR & NR & 2,13 & $\begin{array}{l}\text { Karhagomba et al. (2013); } \\
\text { Kasali et al. (2014b); } \\
\text { Lusakibanza (2012) }\end{array}$ \\
\hline Meliaceae & Azadirachta indica A. Juss. & $\mathrm{T}$ & $\mathrm{Ph}$ & $\mathrm{L}, \mathrm{RB}, \mathrm{SB}$ & $\mathrm{De}, \mathrm{Ma}$ & Oral & 1,3 & $\begin{array}{l}\text { Kasali et al. (2014a); } \\
\text { Chiribagula et al. (2017); } \\
\text { Mbuyi et al. (2019) }\end{array}$ \\
\hline Meliaceae & Entandrophragma palustre Staner & $\mathrm{T}$ & $\mathrm{Ph}$ & SB & De & Oral & 4,12 & $\begin{array}{l}\text { Ngbolua et al. (2014); } \\
\text { Lusakibanza (2012) }\end{array}$ \\
\hline Meliaceae & Khaya nyasica Stapf ex Baker f. & $\mathrm{T}$ & $\mathrm{Ph}$ & SB & $\mathrm{De}$ & Oral & 1,3 & $\begin{array}{l}\text { Kasika et al. (2015); Mbuyi et al. } \\
\text { (2019); Muya et al. (2014) }\end{array}$ \\
\hline Meliaceae & Carapa procera DC. & $\mathrm{T}$ & $\mathrm{Ph}$ & $\mathrm{SB}, \mathrm{Se}$ & $\mathrm{De}$ & Chewing, oral & 6 & Ilumbe Bayeli (2010) \\
\hline Menispermaceae & $\begin{array}{l}\text { Cissampelos owariensis } \\
\text { P. Beauv. ex DC. }\end{array}$ & $\mathrm{L}$ & $\mathrm{Ph}$ & EP & De & NR & 1 & Kalonda et al. (2014) \\
\hline Menispermaceae & Triclisia dictyophylla Diels & L & $\mathrm{Ph}$ & L & $\mathrm{De}$ & Oral & 4 & Ngbolua et al. (2014) \\
\hline Menispermaceae & $\begin{array}{l}\text { Stephania abyssinica } \\
\text { (Quart. -Dill. \& A. Rich.) Walp. }\end{array}$ & $\mathrm{L}$ & $\mathrm{Ph}$ & $L, R, S B$ & De & Enema & 1 & Mbuyi et al. (2019) \\
\hline Menispermaceae & Penianthus longifolius Miers & Sh & $\mathrm{Ph}$ & RB & De & Oral & 4 & Ngbolua et al. (2014) \\
\hline Moraceae & Ficus exasperata Vahl & $\mathrm{T}$ & $\mathrm{Ph}$ & $\mathrm{L}$ & Ma & Oral & 2 & Manya et al. (2020) \\
\hline Moraceae & Ficus thonningii Blume & $\mathrm{T}$ & $\mathrm{Ph}$ & L, RB & $\mathrm{De}$ & Oral & 1 & Mbuyi et al. (2019) \\
\hline Moraceae & Ficus sur Forssk. & Sh & $\mathrm{Ph}$ & L, RB, SB & De & Oral & 1 & Mbuyi et al. (2019) \\
\hline Musaceae & Musa x paradisiaca L. & $\mathrm{H}$ & $\mathrm{Ph}$ & $\mathrm{L}$ & $\begin{array}{l}\text { Leaves roasted } \\
\text { in palm oil }\end{array}$ & Oral & 1 & Mbuyi et al. (2019) \\
\hline Myristicaceae & Pycnanthus marchalianus Ghesq. & $\mathrm{T}$ & $\mathrm{Ph}$ & SB & $\mathrm{De}$ & Oral & 6 & Ilumbe Bayeli (2010) \\
\hline Myrtaceae & Callistemon speciosus (Sims) Sweet & $\mathrm{T}$ & $\mathrm{Ph}$ & $\mathrm{L}$ & $\mathrm{De}$ & NR & 3 & Kasali et al. (2014a) \\
\hline Myrtaceae & $\begin{array}{l}\text { Corymbia citriodora (Hook.) } \\
\text { K.D. Hill \& L.A.S. Johnson }\end{array}$ & $\mathrm{T}$ & $\mathrm{Ph}$ & L & De & $\begin{array}{l}\text { Oral, } \\
\text { inhalation }\end{array}$ & 1 & Mbuyi et al. (2019) \\
\hline Myrtaceae & Eucalyptus globulus Labill. & $\mathrm{T}$ & $\mathrm{Ph}$ & L & De & $\begin{array}{l}\text { Inhalation, } \\
\text { oral }\end{array}$ & $1,2,3$ & $\begin{array}{l}\text { Kasali et al. (2014a); Kasali et } \\
\text { al. (2014b); Mbuyi et al. (2019) }\end{array}$ \\
\hline Myrtaceae & Psidium guajava L. & $\mathrm{T}$ & $\mathrm{Ph}$ & L & De & Oral & $1,2,3$ & $\begin{array}{l}\text { Kasali et al. (2014a); Kasali et } \\
\text { al. (2014b); Mbuyi et al. (2019) }\end{array}$ \\
\hline Myrtaceae & Syzygium guineense (Willd.) DC. & $\mathrm{T}$ & $\mathrm{Ph}$ & $\mathrm{L}, \mathrm{RB}, \mathrm{SB}$ & De & Oral & 1,3 & $\begin{array}{l}\text { Kasali et al. (2014a); } \\
\text { Mbuyi et al. (2019) }\end{array}$ \\
\hline Myrtaceae & Eucalyptus sp. & $\mathrm{T}$ & $\mathrm{Ph}$ & S & $\operatorname{lnf}$ & NR & 3 & Kasali et al. (2014a) \\
\hline Nyctaginaceae & Mirabilis jalapa L. & Sh & $\mathrm{Ch}$ & L & De & NR & 2 & Kasali et al. (2014b) \\
\hline Ochnaceae & Ochna schweinfurthiana F. Hoffm. & Sh & $\mathrm{Ph}$ & $\mathrm{L}, \mathrm{R}$ & $\mathrm{De}, \mathrm{Ma}$ & Oral & 1,2 & $\begin{array}{l}\text { Mbuyi et al. (2019); } \\
\text { Manya et al. (2020) }\end{array}$ \\
\hline Oleaceae & Schrebera trichoclada Welw. & Sh & $\mathrm{Ph}$ & L, S & $\mathrm{De}, \mathrm{Ma}$ & NR & 1 & Kalonda et al. (2014) \\
\hline Passifloraceae & Passiflora edulis Sims & $\mathrm{L}$ & $\mathrm{Ch}$ & L & $\mathrm{De}$ & NR & 3 & Kasali et al. (2014a) \\
\hline Passifloraceae & Passiflora foetida L. & $\mathrm{H}$ & Th & $\mathrm{L}$ & $\operatorname{lnf}$ & NR & 2 & Kasali et al. (2014b) \\
\hline Pentadiplandraceae & Pentadiplandra brazzeana Baill. & Sh & $\mathrm{Ph}$ & $\mathrm{R}, \mathrm{S}$ & $\mathrm{De}$ & Oral & 6,8 & $\begin{array}{l}\text { llumbe Bayeli (2010); } \\
\text { Ndombe et al. (2016) }\end{array}$ \\
\hline
\end{tabular}


Appendix 1 (continued)

\begin{tabular}{|c|c|c|c|c|c|c|c|c|}
\hline Plant family & Botanical name & $\begin{array}{l}\text { Morphological } \\
\text { type }\end{array}$ & $\begin{array}{l}\text { Life } \\
\text { form }\end{array}$ & $\begin{array}{l}\text { Part } \\
\text { used }\end{array}$ & $\begin{array}{l}\text { Preparation } \\
\text { mode }\end{array}$ & $\begin{array}{l}\text { Used } \\
\text { methods }\end{array}$ & Provinces & References \\
\hline Phyllanthaceae & Hymenocardia acida Tul. & Sh & $\mathrm{Ph}$ & $\mathrm{L}$ & De & $\begin{array}{l}\text { Oral, } \\
\text { inhalation, } \\
\text { bath }\end{array}$ & 1 & Mbuyi et al. (2019) \\
\hline Phytolaccaceae & Phytolacca dodecandra L’Hérit. & Sh gr & $\mathrm{Ch}$ & $\mathrm{R}$ & $\mathrm{De}$ & NR & 2 & Kasali et al. (2014b) \\
\hline Picrodendraceae & $\begin{array}{l}\text { Oldfieldia dactylophylla } \\
\text { (Welw. ex Oliv.) J. Léonard }\end{array}$ & $\mathrm{T}$ & $\mathrm{Ph}$ & $\mathrm{RB}, \mathrm{SB}$ & De & Oral, bath & 1 & Mbayo et al. (2016) \\
\hline Piperaceae & $\begin{array}{l}\text { Piper guineense Schum. } \\
\text { and Thonn. }\end{array}$ & $\mathrm{L}$ & $\mathrm{Ph}$ & $\mathrm{L}, \mathrm{Se}$ & De & Oral & 2,6 & $\begin{array}{l}\text { Kasali et al. (2014b); } \\
\text { Ilumbe Bayeli (2010) }\end{array}$ \\
\hline Piperaceae & Piper nigrum L. & $\mathrm{L}$ & Th & $\mathrm{L}$ & NR & NR & 4 & Ngbolua et al. (2015) \\
\hline Piperaceae & Piper capense L.f. & Sh & Th & NR & NR & NR & 2,3 & $\begin{array}{l}\text { Karhagomba et al. (2013); } \\
\text { Kasika et al. (2015) }\end{array}$ \\
\hline Plantaginaceae & Plantago palmata Hook. f. & $\mathrm{H}$ & $\mathrm{Ph}$ & $\mathrm{L}$ & De & Oral & 2 & $\begin{array}{l}\text { Kasali et al. (2014b); } \\
\text { Manya et al. (2020) }\end{array}$ \\
\hline Poaceae & $\begin{array}{l}\text { Cymbopogon densiflorus } \\
\text { (Steud.) Stapf }\end{array}$ & $\mathrm{H}$ & $\mathrm{Ch}$ & AP & De, Inf, Ma & $\begin{array}{l}\text { Inhalation, } \\
\text { oral }\end{array}$ & 1 & $\begin{array}{l}\text { Mbuyi et al. (2019); } \\
\text { Muya et al. (2014) }\end{array}$ \\
\hline Poaceae & Cymbopogon citratus (DC.) Stapf & $\mathrm{H}$ & Th & $\mathrm{L}$ & De, Inf & $\begin{array}{l}\text { Oral, } \\
\text { inhalation, } \\
\text { friction }\end{array}$ & $\begin{array}{l}1,2,3 \\
4,5\end{array}$ & $\begin{array}{l}\text { Ngbolua et al. (2016); Ngbolua } \\
\text { et al. (2019); Mbuyi et al. (2019); } \\
\text { Kasali et al. (2014); Kasali } \\
\text { et al. (2014b); Nzuki (2016) }\end{array}$ \\
\hline Polygalaceae & $\begin{array}{l}\text { Securidaca longipedunculata } \\
\text { Fresen. }\end{array}$ & $\mathrm{T}$ & $\mathrm{Ph}$ & L, SB & De & $\begin{array}{l}\text { Inhalation, } \\
\text { oral }\end{array}$ & 1 & $\begin{array}{l}\text { Mbuyi et al. (2019); } \\
\text { Muya et al. (2014) }\end{array}$ \\
\hline Proteaceae & $\begin{array}{l}\text { Faurea rochetiana (A. Rich.) } \\
\text { Chiov. ex Pic. Serm. }\end{array}$ & $\mathrm{T}$ & $\mathrm{Ph}$ & SB & $\mathrm{De}, \mathrm{Ma}$ & Oral & 1 & Mbuyi et al. (2019) \\
\hline Ranunculaceae & Ranunculus multifidus Forssk. & $\mathrm{H}$ & $\mathrm{Ph}$ & L & De & NR & 3 & Kasali et al. (2014a) \\
\hline Rhamnaceae & $\begin{array}{l}\text { Ziziphus abyssinica Hochst. } \\
\text { ex A. Rich. }\end{array}$ & Sh & $\mathrm{Ph}$ & $\mathrm{R}$ & $\mathrm{Ma}$ & Oral & 1 & Mbuyi et al. (2019) \\
\hline Rhamnaceae & Ziziphus mucronata Willd. & $\mathrm{T}$ & $\mathrm{Ph}$ & $\mathrm{R}$ & $\mathrm{Ma}$ & Oral & 1 & Mbuyi et al. (2019) \\
\hline Rhamnaceae & $\begin{array}{l}\text { Zizyphus resinosa Hochst. } \\
\text { ex A. Rich. }\end{array}$ & $\mathrm{T}$ & $\mathrm{Ph}$ & $\mathrm{R}$ & $\mathrm{De}, \mathrm{Ma}$ & Oral & 1 & Chiribagula et al. (2017) \\
\hline Rosaceae & Prunus africana (Hook. f.) Kalkman & $\mathrm{T}$ & $\mathrm{Ph}$ & B & NR & NR & 2 & Mangambu et al. (2015a) \\
\hline Rosaceae & Rubus rigidus Sm. & L & $\mathrm{Ph}$ & L & $\mathrm{De}$ & NR & 3 & Kasali et al. (2014a) \\
\hline Rubiaceae & $\begin{array}{l}\text { Fadogiella stigmatoloba } \\
\text { (K. Schum.) Robyns }\end{array}$ & $\mathrm{H}$ & $\mathrm{Ch}$ & $\mathrm{L}$ & $\mathrm{De}$ & Oral & 2 & Manya et al. (2020) \\
\hline Rubiaceae & Otiophora pauciflora Baker & $\mathrm{H}$ & $\mathrm{Ch}$ & $\mathrm{L}$ & $\mathrm{Ma}$ & Oral & 2 & Chifundera (2001) \\
\hline Rubiaceae & $\begin{array}{l}\text { Spermacoce princeae (K. Schum.) } \\
\text { Verdc. }\end{array}$ & $\mathrm{H}$ & $\mathrm{Ch}$ & $\mathrm{L}$ & De, Sap & Instillation & 2 & Manya et al. (2020) \\
\hline Rubiaceae & Cinchona calisaya Wedd. & $\mathrm{T}$ & $\mathrm{Ph}$ & B & $\mathrm{De}$ & NR & 2,3 & $\begin{array}{l}\text { Kasali et al. (2014a); } \\
\text { Kasali et al. (2014b) }\end{array}$ \\
\hline Rubiaceae & $\begin{array}{l}\text { Leptactina benguelensis (Welw. } \\
\text { ex Benth \& Hook. f.) R.D. Good. }\end{array}$ & $\mathrm{T}$ & $\mathrm{Ph}$ & L & De & Oral & 4 & Ngbolua et al. (2014) \\
\hline Rubiaceae & Morinda longiflora G. Don & climbing shrub & $\mathrm{Ph}$ & $\mathrm{L}$ & $\mathrm{De}$ & NR & 4 & Makumbelo et al. (2008) \\
\hline Rubiaceae & $\begin{array}{l}\text { Morinda morindoides (Baker) } \\
\text { Milne-Redh. }\end{array}$ & $\mathrm{L}$ & $\mathrm{Ph}$ & $L$ & De & Oral, chewing & $4,5,6$ & $\begin{array}{l}\text { Ngbolua et al. (2013b); Ngbolua } \\
\text { et al. (2016); Ngbolua et al. } \\
\text { (2019); Ngbolua et al. (2014); } \\
\text { Ilumbe Bayeli (2010) }\end{array}$ \\
\hline Rubiaceae & $\begin{array}{l}\text { Rothmannia engleriana } \\
\text { (K. Schum.) Kea }\end{array}$ & $\mathrm{T}$ & $\mathrm{Ph}$ & $L, R B$ & $\mathrm{De}, \mathrm{Ma}$ & Oral & 2 & Manya et al. (2020) \\
\hline Rubiaceae & Cinchona officinalis L. & $\mathrm{T}$ & $\mathrm{Ph}$ & $\mathrm{L}, \mathrm{SB}$ & De & Oral & 2 & Manya et al. (2020) \\
\hline Rubiaceae & Morinda lucida Benth. & Sh & $\mathrm{Ph}$ & L, UP & $\mathrm{De}$ & Oral, friction & 4,5 & $\begin{array}{l}\text { Ngbolua et al. (2019); } \\
\text { Nzuki (2016) }\end{array}$ \\
\hline Rubiaceae & $\begin{array}{l}\text { Gardenia ternifolia Schumach. } \\
\text { \& Thonn. }\end{array}$ & Sh & $\mathrm{Ph}$ & $\mathrm{R}$ & De & Oral & 1 & Mbuyi et al. (2019) \\
\hline Rubiaceae & $\begin{array}{l}\text { Hymenodictyon floribundum } \\
\text { (Hoscht. \& Steud) B.L. Rob. }\end{array}$ & Sh & $\mathrm{Ph}$ & $\mathrm{R}$ & $\mathrm{De}$ & NR & 1 & Kalonda et al. (2014) \\
\hline Rubiaceae & $\begin{array}{l}\text { Sarcocephalus latifolius (Sm.) } \\
\text { E.A. Bruce }\end{array}$ & Sh & $\mathrm{Ph}$ & $\mathrm{R}$ & $\mathrm{De}$ & NR & 5 & Ngbolua et al. (2013a) \\
\hline Rubiaceae & $\begin{array}{l}\text { Crossopteryx febrifuga (Afzel. } \\
\text { ex G. Don) Benth. }\end{array}$ & $\mathrm{T}$ & $\mathrm{Ph}$ & SB & $\mathrm{De}$ & Oral & 1 & $\begin{array}{l}\text { Mbuyi et al. (2019); } \\
\text { Kalonda et al. (2014) }\end{array}$ \\
\hline Rubiaceae & $\begin{array}{l}\text { Nauclea diderrichii } \\
\text { (De Wild.) Merr. }\end{array}$ & Sh & $\mathrm{Ph}$ & SB & De & Oral & 4 & Ngbolua et al. (2014) \\
\hline Rutaceae & Citrus aurantium L. & $\mathrm{T}$ & $\mathrm{Ph}$ & $\mathrm{Fr}$ & $\mathrm{Ma}$ & NR & 2 & Kasali et al. (2014b) \\
\hline Rutaceae & Citrus limon (L.) Osbeck & $\mathrm{T}$ & $\mathrm{Ph}$ & $\mathrm{L}$ & $\mathrm{De}$ & Oral & $1,2,3$ & $\begin{array}{l}\text { Kasali et al. (2014a); Kasali et } \\
\text { al. (2014b); Mbuyi et al. (2019) }\end{array}$ \\
\hline Rutaceae & Citrus medica L. & $\mathrm{T}$ & $\mathrm{Ph}$ & $\mathrm{L}$ & Inf & Bath & 9 & Mbula et al. (2015) \\
\hline Sapindaceae & Zanha africana (Radlk.) Exell & $\mathrm{T}$ & $\mathrm{Ph}$ & RB & Inc & Tatoo & 1 & Mbuyi et al. (2019) \\
\hline Sapotaceae & $\begin{array}{l}\text { Autranella congolensis (De Wild.) } \\
\text { A. Chev. }\end{array}$ & $\mathrm{T}$ & $\mathrm{Ph}$ & SB & De & Oral & 4 & Ngbolua et al. (2014) \\
\hline
\end{tabular}


Bois et Forêts des Tropiques - ISSN: L-0006-579X

Volume $347-1^{\text {st }}$ quarter - February $2021-$ p. 11-27

24 FOCUS / ANTIMALARIAL MEDICINAL PLANTS

\section{Appendix 1 (continued)}

\begin{tabular}{|c|c|c|c|c|c|c|c|c|}
\hline Plant family & Botanical name & $\begin{array}{l}\text { Morphological } \\
\text { type }\end{array}$ & $\begin{array}{l}\text { Life } \\
\text { form }\end{array}$ & $\begin{array}{l}\text { Part } \\
\text { used }\end{array}$ & $\begin{array}{l}\text { Preparation } \\
\text { mode }\end{array}$ & $\begin{array}{l}\text { Used } \\
\text { methods }\end{array}$ & Provinces & References \\
\hline Simaroubaceae & Quassia Africana (Baill.) Baill. & $\mathrm{T}$ & $\mathrm{Ph}$ & $L, R$ & NR & Oral & 4 & $\begin{array}{l}\text { Ngbolua et al. (2015); } \\
\text { Ngbolua et al. (2016) }\end{array}$ \\
\hline Solanaceae & Solanum sisymbriifolium Lam. & $\mathrm{H}$ & $\mathrm{Ch}$ & $\mathrm{Fr}$ & Inf & $\begin{array}{l}\text { Oral/reactally } \\
\text { applied }\end{array}$ & 2 & Chifundera (2001) \\
\hline Solanaceae & Capsicum annuum L. & $\mathrm{T}$ & $\mathrm{Ch}$ & L & $\mathrm{Ma}, \operatorname{lnf}$ & NR & 1 & Kalonda et al. (2014) \\
\hline Solanaceae & Solanum incanum L. & $\mathrm{H}$ & $\mathrm{Ch}$ & $\mathrm{R}$ & $\mathrm{De}, \mathrm{Ma}$ & Enema & 1 & Mbuyi et al. (2019) \\
\hline Solanaceae & Nicotiana tabacum L. & $\mathrm{H}$ & Th & L & Gri & Noise & 1 & Mbuyi et al. (2019) \\
\hline Solanaceae & Physalis peruvianna L. & $\mathrm{H}$ & Th & $\mathrm{L}, \mathrm{R}$ & De, Inf & Oral & $1,2,3$ & $\begin{array}{l}\text { Kasali et al. (2014a); Kasali et } \\
\text { al. (2014b); Mbuyi et al. (2019); } \\
\text { Manya et al. (2020) }\end{array}$ \\
\hline Solanaceae & Physalis angulata L. & $\mathrm{H}$ & Th & WP & NR & NR & 5 & Lusakibanza Manzo (2012) \\
\hline Tropaeolaceae & Tropaeolum majus L. & $\mathrm{H}$ & $\mathrm{Ch}$ & $\mathrm{L}$ & De & NR & 2,3 & $\begin{array}{l}\text { Kasali et al. (2014a); } \\
\text { Kasali et al. (2014b) }\end{array}$ \\
\hline Verbenaceae & Lantana camara L. & Sh & $\mathrm{Ph}$ & $\mathrm{L}$ & De, Inf & $\begin{array}{l}\text { Inhalation, } \\
\text { oral }\end{array}$ & $1,2,3$ & $\begin{array}{l}\text { Kasali et al. (2014a); Kasali et } \\
\text { al. (2014b); Mbuyi et al. (2019); } \\
\text { Manya et al. (2020) }\end{array}$ \\
\hline Verbenaceae & Lippia multiflora Moldenke & $\mathrm{H}$ & & $\mathrm{L}$ & De & Oral & 5 & Nzuki (2016) \\
\hline Zingiberaceae & Curcuma longa L. & $\mathrm{H}$ & $\mathrm{Ge}$ & L & $\mathrm{Ma}$ & Oral & 5 & Nzuki (2016) \\
\hline Zingiberaceae & Aframomum melegueta K. Schum. & $\mathrm{H}$ & $\mathrm{Ge}$ & $L, R$ & De & Oral & 1 & Mbuyi et al. (2019) \\
\hline Zingiberaceae & $\begin{array}{l}\text { Aframomum alboviolaceum } \\
\text { (Ridl.) K. Schum. }\end{array}$ & $\mathrm{H}$ & $\mathrm{Ge}$ & $\mathrm{L}, \mathrm{R}, \mathrm{Fr}$ & $\mathrm{Ma}$ & Oral & 4,6 & $\begin{array}{l}\text { Ngbolua et al. (2016); } \\
\text { Ngbolua et al. (2019); } \\
\text { Illumbe Bayeli (2010) }\end{array}$ \\
\hline Zingiberaceae & $\begin{array}{l}\text { Aframomum laurentii (De Wild. } \\
\text { \& T. Durand) K. Schum. }\end{array}$ & $\mathrm{H}$ & $\mathrm{Ph}$ & $\mathrm{L}$ & $\begin{array}{l}\text { De, Inf, } \\
\text { powder }\end{array}$ & $\begin{array}{l}\text { Oral, topical } \\
\text { application }\end{array}$ & 2 & Manya et al. (2020) \\
\hline
\end{tabular}

Morphological type (H: herb, T: tree, Sh: shrub, Ssh: sub-shrub, L: liana), life-form (Ph: Phanerophyte, Ch: Chamaephyte, Hc: Hemicryphophyte, Th: Therophyte, Ge: Geophyte), part used (L: leaves, R: root, RB: root bark, WP: whole plant, Fr: fruit, AP: arial part, B: bark, Gr: grain, Se: seed, Fl: flower, S: stem, SB: stem bark, UP: underground part, EP: external part), preparation mode (De: decoction, Ma: maceration, Inf: infusion, Gri: grinding, Exp: expression, Ext: extorsion, Inc: incineration), Provinces (1: Haut-Katanga, 2: Sud-Kivu, 3: Nord-Kivu, 4: Kinshasa, 5: Kongo-Central, 6: Equateur, 7: Ituri, 8: Kwango, 9: Tshopo, 10: Sud-Ubangi, 11: Lualaba, 12: Sankuru, 13: Maniema).

\section{References}

Adekunle M. F., 2008. Indigenous uses of plant leaves to treat malaria fever at Omo forest reserve (OFR) Ogun State, Nigeria. Ethiopian Journal of Environmental Studies and Management, 1 (1): 31-35. https://doi. org/10.4314/ejesm.v1i1.41567

Adia M. M., Anywar G., Byamukama R., Mugisha M. K., Sekagya Y., Kakudidi E K., et al., 2014. Medicinal plants used in malaria treatment by Prometra herbalists in Uganda. Journal of Ethnopharmacology, 155: 580588. https://doi.org/10.1016/j.jep.2014.05.060

Alalwan T. A., Alkhuzai J. A., Jameel Z., Mandeel Q. A., 2019. Quantitative ethnobotanical study of some medicinal plants used by herbalists in Bahrain. Journal of Herbal Medicine, 17-18: 100278. https://doi. org/10.1016/j.hermed.2019.100278

Alebie G., Urga B., Worku A., 2017. Systematic review on traditional medicinal plants used for the treatment of malaria in Ethiopia: trends and perspectives. Malaria Journal, 16: 307. https://doi.org/10.1186/ s12936-017-1953-2

Alker A. P., Kazadi W. M., Kutelemeni A. K., Bloland P. B., Tshefu A. K., Meshnick S. R., 2008. Dhfr and dhps genotype and sulfadoxine-pyrimethamine treatment failure in children with falciparum malaria in the Democratic Republic of Congo. Tropical Medicine and International Health, 13 (11): 1384-1391. https://doi.org/10.1111/j.13653156.2008.02150.x

Amjad M. S., Qaeem M. F., Ahmad I., Khan S. U., Chaudhari S. K., Zhaid M. N., et al., 2017. Descriptive study of plant resources in the context of the ethnomedicinal relevance of indigenous flora: A case study from Toli Peer National Park, Azad Jammu and Kashmir, Pakistan. PLoS ONE, 12 (2): e0171896. https://doi.org/10.1371/journal.pone.0171896
Anywar G., Van't Klooster C. E. I. A., Byamukama R., Willcox M., Nalumansi P. A., De Jong J., et al., 2016. Medicinal plants used in the treatment and prevention of malaria in Cegere Sub-County, Northern Uganda. Ethnobotany Research and Applications, 14: 505-516. http://dx.doi. org/10.17348/era.14.0.505-516

APG, 2009. An update of the Angiosperm phylogeny group classification for the orders and families of flowering plants: APGIII. Botanical Journal of the Linnean Society, 161: 105-121. https://doi.org/10.1111/j.10958339.2009.00996.x

Arshad M., Ahmad M., Ahmed E., Saboor A., Abbas A., Sadiq S., 2014. An ethnobiological study in Kala Chitta hills of Pothwar region, Pakistan: multinomial logit specification. Journal of Ethnobiology and Ethnomedicine, 10: 13. http://www.ethnobiomed.com/content/10/1/13

Asadi-Samani M., Rafieian-Kopaei M., Azimi N., 2013. Gundelia: A systematic review of medicinal and molecular perspective. Pakistan Journal of Biological Sciences, 16 (21): 1238-1247. https://doi.org/10.3923/ pjbs.2013.1238.1247

Asase A., Akwetey G. A., Achel D. G., 2010. Ethnopharmacological use of herbal remedies for the treatment of malaria in the Dagme West District of Ghana. Journal of Ethnopharmacology, 129: 367-376. https://doi. org/10.1016/i.jep.2010.04.001

Bakwaye F. N., Termote C., Kimbungu K., Van Damme P., 2013. Identification et importance locale des plantes médicinales utilisées dans la région de Mbanza-Ngungu, République démocratique du Congo. Bois et Forêts des Tropiques, 316 (2) : 63-77. https://doi.org/10.19182/ bft2013.316.a20531

Balima L. H., Nacoulma B. M. I., Ekué M. R. M., Kouamé F. N., Thiombiano A., 2018. Use patterns, use values and management of Afzelia africana $\mathrm{Sm}$. in Burkina Faso: implications for species domestication and sustainable conservation. Journal of Ethnobiology and Ethnomedicine, 14: 23. https://doi.org/10.1186/s13002-018-0221-z 
Betti J. L., Caspa R., Ambara J., Kourogue R. L., 2013a. Ethno-botanical study of plants used for treating malaria in a forest: savanna margin area, East region, Cameroon. Global Journal of Research on Medicinal Plants and Indigenous Medicine, 2 (10): 692-708. http://www.ethnopharmacologia.org/prelude2020/pdf/biblio-hb-56-betti.pdf

Betti J. L., Iponga D. M., Yongo O. D., Mbomio D. O., Yobo C. M., Ngoy A., 2013b. Ethnobotanical study of medicinal plants of the Ipassa-Makokou Biosphere, Gabon: Plants used for treating malaria. Journal of Medicinal Plants Research, 7 (31): 2300-2318. https://doi.org/10.5897/ IMPR12.1211

Betti J. L., 2002. Medicinal plants sold in Yaoundé markets, Cameroon. African Study Monographs, 23 (2): 47-64. https://citeseerx.ist.psu.edu/ viewdoc/download?doi=10.1.1.574.8891\&rep=rep1\&type=pdf

Bibi T., Ahmad M., Tareen N. M., Jabeen R., Sultana S., Zafar M., et al., 2015. The endemic medicinal plants of Northern Balochistan, Pakistan and their uses in traditional medicine. Journal of Ethnopharmacology, 173: 1-10. https://doi.org/10.1016/i.jep.2015.06.050

Bora U., Sahu A., Saikia A. P., Ryakala V. K., Goswami P., 2007. Medicinal plants used by the people of Northeast India for curing malaria. Phytotherapy Research, 21: 800-804. https://doi.org/10.1002/ptr.2178 Chifundera K., 2001. Contribution to the inventory of medicinal plants from the Bushi area, South Kivu Province, Democratic Republic of Congo. Fitoterapia, 72: 351-386. https://doi.org/10.1016/s0367326x(00)00294-X

Chiribagula V. B., Amuri S. B., Kalonji S. M., Byanga J. K., Duez P., Simbi J. B. L., 2017. Étude ethnobotanique, phytochimique et évaluation de l'activité antiplasmodiale de 13 plantes réputées antipaludéennes dans la commune du Kenya (Lubumbashi, RDC). Phytothérapie, https://doi. org/10.1007/s10298-017-1152-x

Faruque M. O., Feng G., Khan Md. N. A., Barlow J. W., Ankhi U R.., Hu S., et al., 2019. Qualitative and quantitative ethnobotanical study of the Pangkhua community in Bilaichari Upazilla, Rangamati District, Bangladesh. Journal of Ethnobiology and Ethnomedicine, 15: 8. https://doi. org/10.1186/s13002-019-0287-2

Gomez Marín E., Merino Cristóbal L., 1990. Plantas Medicinales de Guinea Ecuatorial. Malabo, Guinea Ecuatorial, Centro cultural Hispano-Guineano ediciones, 262 p. https://core.ac.uk/download/pdf/158825712.pdf

Idowu O. A., Soniran O. T., Ajana O., Aworinde D. O., 2010. Ethnobotanical survey of antimalarial plants used in Ogun State, Southwest Nigeria. African Journal of Pharmacy and Pharmacology, 4 (2): 055-060. https://academicjournals.org/article/article1380869467_Idowu\%20et\%20al.pdf

Ilumbe Bayeli G., 2010. Utilisation des plantes en médecine traditionnelle par les Pygmées (Ba-Twa) et les Bantous (Ba-Oto) du territoire de Bikoro, Province de l'Équateur en République Démocratique du Congo. Thèse de doctorat, Université libre de Bruxelles, Belgique. https://difusion.ulb.ac.be/vufind/Record/ULB-DIPOT:oai:dipot.ulb. ac.be:2013/210007/TOC

IUCN, 2019. The IUCN Red List of Threatened Species. http://www. iucnredlist.org

Iyamah P. C., Idu M., 2015. Ethnomedicinal survey of plants used in the treatment of malaria in Southern Nigeria. Journal of Ethnopharmacology, 173: 287-302. https://doi.org/10.1016/j.jep.2015.07.008

Kamatenesi-Mugisha M., Oryem-Origa H., 2005. Traditional herbal remedies used in the management of sexual impotence and erectile dysfunction in western Uganda. African Health Sciences, 5 (1): 40-49. https:// www.ajol.info/index.php/ahs/article/view/6896

Kami T., Moustsambote J.-M., Nzobadila E. K., Atencia R., 2019. Floristic and phytogeographical analysis at east Conkouati-Douli National Park (Republic of Congo). International Journal of Innovation and Applied Studies, 27 (1): 122-132. https://www.semanticscholar.org/paper/Floristic-and-phytogeographycal-analysis-at-east-of-Kami-Moutsambote/ c7f835cb35d45f53d1f3a2217267943b637dbae7

Kalonda E. M., Mbayo M., Muhume S. K., Kasereka M., Mulamba J. M., Mboni M., et al., 2014. Ethnopharmacological survey of plants used against malaria in Lubumbashi city (D.R. Congo). Journal of Advanced Botany and Zoology, 1 (2): 1-8. https://pdfs.semanticscholar.org/ dcf2/21824f3d63d28188196d7b18be0b7ab0e30c.pdf

Karhagomba I. B., Mirindi T. A., Mushagalusa T. B., Nabino V. B., Koh K., Kim H. S., 2013. The cultivation of wild food and medicinal plants for improving community livelihoods: The case of the Buhozi site, DR Congo. Nutrition Research and Practice, 7 (6): 510-518. https://doi. org/10.4162/nrp.2013.7.6.510
Kasali F. M., Mahano A. O., Kadima N. J., Mpiana P. T., Ngobolua K. N., Tshibangu T. S. D., 2014a. Ethnopharmacological survey of medicinal plants used against malaria in Butembo city (D.R. Congo). Journal of Advanced Botany and Zoology, 1 (1): 1-11.

Kasali F. M., Mahano A. O., Nyakabwa D. S., Kadima N. J., Misakabu F. M., Tshibangu D. S. T., et al., 2014b. Ethnopharmacological survey of medicinal plants used against malaria in Bukavu city (D.R. Congo). European Journal of Medicinal Plants, 4 (1): 29-44. https://doi.org/10.9734/ EJMP/2014/5766

Kasika E. L., Vasombolwa V. K., Lejoly J., 2015. Contribution to the knowledge of plants used by Bantu and Pygmy healers in Beni and Lubero territories (Democratic Republic of Congo). Journal of Plant Studies, 4 (2): 157-176. https://doi.org/10.5539/ips.v4n2p157

Kayani S., Ahmad M., Zafar M., Sultana S., Khan M. P. Z., Ashraf M. A., et $a l, 2014$. Ethobotanical uses of medicinal plants for respiratory disorders among the inhabitants of Gallies-Abbottabad, Northern Pakistan. Journal of Ethnopharmacology, 156: 47-60. https://doi.org/10.1016/i. iep.2014.08.005

Kikufi A., Lukoki F., 2008. Étude floristique et écologique des marais de Masina. Revue Congolaise des Sciences Nucléaires, 23 (1) : 1-19. https://www.academia.edu/8827362/ETUDE_FLORISTIQUE_ET_ECOLOGIQUE DES MARAIS DE MASINA

Kikufi A., Lejoly J., Lukoki F., 2017. État actuel de la biodiversité végétale du territoire de Kimvula au sud-ouest de la République Démocratique du Congo. International Journal of Innovation and Applied Studies, 19 (4) : 929-943. https://studylibfr.com/doc/426261/\%C3\%A9tat-actuel-de-la-biodiversit\%C3\%A9-v\%C3\%A9g\%C3\%A9tale-du-territoire Kodi P., Mwangi E. M., Cheplogoi P. K., Kariruki T. S., 2017. Ethnobotanical survey of antimalarial medicinal plants used in Butebo County, Eastern Uganda. European Journal of Medicinal Plants, 21 (4): 1-22. https:// doi.org/10.9734/EJMP/2017/35368

Konda M., Kabakura M., Mbembe B., Itufa O., Mahuku K., Mafuta M. et al., 2012. Plantes médicinales de traditions, Province de l'Équateur - R.D. Congo. Ouagadougou, Burkina Faso, Institut de recherche en sciences de la santé (IRSS), 420 p. http://www.ethnopharmacologia. org/prelude2018/pdf/biblio-hk-60-konda-ku-mbuta.pdf

Koudouvo K., Karou D. S., Kokou K., Essien K., Aklikokou K., Glitho I. A., et al., 2011. An ethnobotanical study of antimalarial plants in Togo Maritime Region. Journal of Ethnopharmacology, 134: 183-190. https://doi. org/10.1016/i.jep.2010.12.011

Kuete V., Efferth T., 2010. Cameroonian medicinal plants: pharmacology and derived natural products. Frontiers in Pharmacology, 1: 1-19. https://doi.org/10.3389/fphar.2010.00123

Lakouéténé D. P. B., Ndolngar G., Berké B., Moyen J.-M., Kosh Komba E., Zinga I., Silla S., et al., 2009. Enquête ethnobotanique des plantes utilisées dans le traitement du paludisme à Bangui. Bulletin de la Société de Pharmacie de Bordeaux, 148 : 123-138. https://studylibfr. $\mathrm{com} / \mathrm{doc} / 887435 /$ enqu\%C3\%AAte-ethnobotanique-des-plantes-utilis\%C3\%A9es-dans-le-trai...

Landis S. H., Lokomba V., Ananth C. V., Atibu J., Ryder R. W., Hartmann K. E., et al., 2009. Impact of maternal malaria and under-nutrition on intrauterine growth restriction: a prospective ultrasound study in Democratic Republic of Congo. Epidemiology and Infection, 137: 294-304. https:// doi.org/10.1017/S0950268808000915

Lassa Kanda L., Kikufi Batoba A., Ilumbe Bayeu G., Biloso Moyene A., Masens Da Musa M., Habari Mulawa J.-P., et al., 2019. Étude floristique, écologique et phytogéographique des espèces utiles du territoire de Kimvula, R. D. Congo. Congo Sciences, 7 (2) : 91-101. http://congosciences. cd/images/vol7-juillet2019/LASSA 2019 Vol 7 N 2 Art 4.pdf

Light J. E., Nessner C. E., Gustafsson D. R., Wise S. R., Voelker G., 2016. Remarkable levels of avian louse (Insecta: Phthiraptera) diversity in the Congo Basin. Zoologica Scripta, 45: 538- 551. https://doi.org/10.1111/ zsc. 12170

Lusakibanza Manzo M., 2012. Étude phytochimique et pharmacologique de plantes antipaludiques utilisées en médecine traditionnelle congolaise. Thèse de doctorat, Université de Liège, Belgique, 223 p. http://bictel.ulg.ac.be/ETD-db/collection/available/ULgetd-07032012-191612/ unrestricted/theseLusakibanza.pdf

Madureira M. C., Martins A. P., Gomes M., Paiva J., Cunha A. P., Rosário V., 2002. Antimalarial activity of medicinal plants used in traditional medicine in S. Tomé and Príncipe islands. Journal of Ethnopharmacology, 81: 23-29. https://doi.org/10.1016/S0378-8741(02)00005-3 
Mahomoodally M. F., 2013. Traditional medicines in Africa: An appraisal of ten potent African medicinal plants. Evidence-Based Complementary and Alternative Medicine, 2013: 1-14. https://doi. org/10.1155/2013/617459

Makumbelo E., Lukoki L., Paulis J. J. S. J., Luyindula N., 2008. Stratégie de valorisation des espèces ressources des produits non ligneux de la savane des environs de Kinshasa. II. Enquête ethnobotanique (aspects médicinaux). Tropicultura, 26 (3) : 129-134. http://www.tropicultura. org/text/v26n3/129.pdf

Mamadou A. J., Douma S., Inoussa M. M., Moussa S., Mahamane A., Saadou M., 2019. Diversity, life forms, chorology and uses of spontaneous medicinal plants in Niamey and Tillabéri Regions, Niger Republic. Journal of Applied Life Sciences International, 22 (4): 1-17. https:// doi.org/10.9734/jalsi/2019/v22i430134

Mangambu M. J. D., Aluma K. J.-Y., Diggelen R. V., Rugenda-Banga R. A. D., Mushangalusa K. F., Chibembe S. A., et al., 2015a. Études ethnobotanique et ethnolinguistique des ressources forestières ligneuses utilisées par la population du couloir écologique du Parc National de Kahuzi-Biega (RD Congo). European Scientific Journal, 11 (15) : 135-162. https://core.ac.uk/download/pdf/236412252.pdf

Mangambu M. J. D., Diggelen R. V., Mwanga Mwanga J. C., Ntahobavuka H., Malaisse F., Robbrecht E., 2012. Étude ethnoptéridologique, évaluation des risques d'extinction et stratégies de conservation aux alentours du Parc National de Kahuzi Biega (RD Congo). Geo-Eco-Trop, 36 : 137-158. http://www.geoecotrop.be/uploads/publications/pub 361 09.pdf

Mangambu M. J. D., Kavatsurwa M. S., Birhashirwa N. R., Habimana N. H., 2015b. Utilisation des ressources forestières ligneuses par la population habitant la zone submontagnarde du Parc National de Kauzi-Biega (R.D. Congo). International Journal of Innovation and Applied Studies, 11 (2) : 508-521. http://www.issr-journals.org/links/papers.php?journal=iiias\&application=pdf\&article=|J|AS-15-094-09

Manya M. H., Keymeulen F., Ngezahayo J., Bakari A. S., Kalonda M. E., Kahumba B. J., et al., 2020. Antimalarial herbal remedies of Bukavu and Uvira areas in DR Congo: An ethnobotanical survey. Journal of Ethnopharmacology, 249: 112422. https://doi.org/10.1016/i.jep.2019.112422 Masharabu T., Noret N., Lejoly J., Bigendako M. J., Bogaert J., 2010. Étude comparative des paramètres floristiques du Parc National de la Ruvubu, Burundi. Geo-Eco-Trop, 34 : 29-44. http://www.geoecotrop.be/uploads/ publications/pub 341 02.pdf

Masunda A. T., Inkoto C. L., Bongo G. N., Oloko J. D. O., Ngbolua K. N., Tshibangu D. S., et al., 2019. Ethnobotanical and ecological studies of plants used in the treatment of Diabetes in Kwango, Kongo Central and Kinshasa in Democratic Republic of the Congo. International Journal of Diabetes and Endocrinology, 4 (1): 18-25. http://www.sciencepublishinggroup.com/iournal/paperinfo?journalid=278\&doi=10.11648/i. ijde.20190401.14

Mbala M. S., 2003. Situation des ressources génétiques forestières de la République démocratique du Congo. Note thématique sur les ressources génétiques forestières. Document de travail FGR/56F. Département des forêts, Division des ressources forestières. FAO, Rome, 48 p. http://www.fao.org/forestry/12467-0ba027e46ab020c197c65d1c023a230a7.pdf

Mbatchi S. F., Mbatchi B., Banzouzi J. T., Bansimba T., Nsonde Ntandou G. F., Ouamba J.-M., et al., 2006. In vitro antiplasmodial activity of 18 plants used in Congo Brazzaville traditional medicine. Journal of Ethnopharmacology, 104 (1-2): 168-174. https://doi.org/10.1016/i. iep.2005.08.068

Mbayo K. M., Kalonda M. E., Tshisand T. P., Kisimba K. E., Mulamba M., Kalunga R. M., et al., 2016. Contribution to ethnobotanical knowledge of some Euphorbiaceae used in traditional medicine in Lubumbash and its surroundings (DRC). Journal of Advanced Botany and Zoology, 4 (2): 1-16. https://www.researchgate.net/profile/Marsi-Mbayo/publication/304806040_Contribution to ethnobotanical_knowledge of some Euphorbiaceae used in traditional medicine in Lubumbashi and its surroundings DRC/links/577bb8a508ae355e74f16071/ Contribution-to-ethnobotanical-knowledge-of-some-Euphorbia ceae-used-in-traditional-medicine-in-Lubumbashi-and-its-surroundings-DRC.pdf

Mbula J. P., Kwembe J. T. K., Tshilanda D. D., Asimonio J., Toegaho A., Tshibangu D. S. T., et al., 2015. Ethnobotanical survey of aromatic plants of Masako Forest Reserve (Kisangani, DR Congo). Journal of Advanced Botany and Zoology, 2 (3): 1-9. http://cd.chm-cbd.net/implementation/centre-de-sureveillance-de-la-biodiversite-csb/botanique/ethnobotanique/ethnobotanical-survey-aromatic-plants-masako-forest-reserve-kisangani-dr-congo
Mbuyi K. S., Kalunga M. R., Kalonda M. E., Cimanga C. C. B., Numbi I. E., Kahumba B. J., et al., 2019. Aperçu ethnobotanique de plantes réputées antipaludéennes utilisées dans la ville de Lubumbashi et ses environs, dans le Haut-Katanga en RD Congo. Ethnopharmacologia, 61 : 75-84. http://www.ethnopharmacologia.org/wp-content/uploads/2019/07/ ETHNOPHARM-61-Mbuyi.pdf

Messina J. P., Taylor S. M., Meshnick S. R., Linke A. M., Tshefu A. K., Atua B., et al., 2011. Population, behavioural and environmental drivers of malaria prevalence in the Democratic Republic of Congo. Malaria Journal, 10: 161. https://doi.org/10.1186/1475-2875-10-161

Mobula L., Lilley B., Tshefu A. K., Rosenthal P. J., 2009. Resistance-mediating Polymorphisms in Plasmodium falciparum infections in Kinshasa, Democratic Republic of the Congo. American Society of Tropical Medicine and Hygiene, 80 (4): 555-558. https://doi.org/10.4269/ ajtmh.2009.80.555

Mongeke M. M., Ngbolua K. N., Bakola R. D., Inkoto C. L., Elikandani P. N., Mouli C. Y., 2018. Enquête sur les plantes utilisées en médicine traditionnelle par les Bambenga : Pygmées du secteur de Dongo en République Démocratique du Congo. Revue Marocaine des Sciences Agronomiques et Vétérinaires, 6 (4) : 469-475. https://www.agrimaroc.org/ index.php/Actes IAVH2/article/view/617

Muganga R., Angenot L., Tits M., Frédérich M., 2010. Antiplasmodial and cytotoxic activities of Rwandan medicinal plants used in the treatment of malaria. Journal of Ethnopharmacology, 128: 52-57. https://doi. org/10.1016/i.jep.2009.12.023

Muya K., Tshoto K., Cioci C. C., Aseho M. M., Kalonji M., Byanga K., et al., 2014. Survol ethnobotanique de quelques plantes utilisées contre la schistosomiase urogénitale à Lubumbashi et environs. Phytothérapie, 12 : 213-228. https://doi.org/10.1007/s10298-014-0877-z

Mvumbi D. M., Kayembe J.-M., Situakibanza H., Bobanga T. L., Nsibu C. N., Mvumbi G. L., et al., 2015. Falciparum malaria molecular drug resistance in the Democratic Republic of Congo: a systematic review. Malaria Journal, 14: 354. https://doi.org/10.1186/s12936-015-0892-z

Ndjele M. B., 1988. Les éléments phytogéographiques endémiques dans la flore vasculaire du Zaïre. Thèse de doctorat, Université libre de Bruxelles, Belgique, $536 \mathrm{p}$.

Ndombe F. M., Ngbolua K. N., Masens B. Y. M., Mpiana P. T., 2016. Études ethnobotanique et écologique des plantes utilisées dans le traitement de la stérilité à Kenge et ses environs (Province du Kwango, République Démocratique du Congo). International Journal of Innovation and Scientific Research, 26 (2) : 600-611. http://www.ijisr.issr-journals.org/abstract.php?article=IJISR-16-233-02

Ngarivhume T., Van't Klooster C. I. E. A., De Jong J. T. V. M., Van der Westhuizen J. H., 2015. Medicinal plants used by traditional healers for the treatment of malaria in the Chipinge district in Zimbabwe. Journal of Ethnopharmacology, 159: 224-237. https://doi.org/10.1016/j. jep.2014.11.011

Ngbolua K. N., Benamambote B. M., Mpiana P. T., Muanda D. M., Ekutsu E., Tshibangu D. S. T., et al., 2013a. Ethno-botanical survey and ecological study of some medicinal plants species traditionally used in the District of Bas-Fleuve (Bas-Congo Province, Democratic Republic of Congo). Research Journal of Chemistry, 1 (2): 1-10. https://www. researchgate. net/publication/258167541_Ethno-botanical_survey_and_Ecological Study of some Medicinal Plants species traditionally used in the District of Bas-Fleuve Bas-Congo Province Democratic Republic of Congo

Ngbolua K. N., Mudogo V., Mpiana P. T., Malekani M. J., Rafatro H., Ratsimamanga S. U., et al., 2013b. Évaluation de l'activité antidrépanocytaire et antipaludique de quelques taxons végétaux de la République démocratique du Congo et de Madagascar. Ethnopharmacologie, 50 : 7-12. Ngbolua K. N., Inkoto C. L., Mongo N. L., Ashande C. M., Masens Y. B., Mpiana P. T., 2019. Étude ethnobotanique et floristique de quelques plantes médicinales commercialisées à Kinshasa, République Démocratique du Congo. Revue Marocaine des Sciences Agronomiques et Vétérinaires, 7 (1) : 118-128. https://core.ac.uk/download/pdf/230580865.pdf Ngbolua K. N., Mandjo L. B., Munsebi M. J., Ashande C. M., Moke E. L., Asamboa S. L., et al., 2015. Études ethnobotanique et écologique des plantes utilisées en médecine traditionnelle dans le District de la Lukunga à Kinshasa (RD du Congo). International Journal of Innovation and Scientific Research, 26 (2) : 612-633. http://www.ijisr.issr-journals. org/abstract.php?article=||ISR-16-233-03 
Ngbolua K. N., Mpiana P. T., Mudogo V., Ngombe N. K., Tshibangu D. S. T., Ekutsu E., et al., 2014. Ethno-pharmacological survey and floristical study of some medicinal plants traditionally used to treat infectious and parasitic pathologies in the Democratic Republic of Congo. International Journal of Medicinal Plants, 106: 454-467.

Ngbolua K. N., Mihigo S. O., Liyongo C. I., Ashande M. C., Tshibangu D. S. T., Zoawe B. G., et al., 2016. Ethno-botanical survey of plant species used in traditional medicine in Kinshasa city (Democratic Republic of the Congo). Tropical Plant Research, 3 (2): 413-427. https://www.tropicalplantresearch.com/archives/2016/vol3issue2/24.pdf

Nsonde-Ntandou G. F., Ndounga M., Ouamba J. M., Gbeassor M., EtouOssebi A., Ntoumi F., et al., 2005. Enquête ethnobotanique : screening chimique et efficacité thérapeutique de quelques plantes utilisées contre le paludisme en médicine traditionnelle à Brazzaville. Phytothérapie, 3 : 13-18. https://doi.org/10.1007/s10298-005-0059-0

Ntie-Kang F., Onguéné P. A., Lifongo L. L., Ndom J. C., Sippl W., Meva'a Mbaze L., 2014. The potential of anti-malarial compounds derived from African medicinal plants. Part II: A pharmacological evaluation of non-alkaloids and non-terpenoids. Malaria Journal, 13: 81. https://doi. org/10.1186/1475-2875-13-81

Nzuki B. F., 2016. Recherches ethnobotaniques sur les plantes médicinales dans la région de Mbanza-Ngungu, RDC. Thèse de doctorat (PhD), Université de Gand, Belgique, 349 p. https://biblio.ugent.be/publication/8205211/file/8205212

Okello D., Kang Y., 2019. Exploring antimalarial herbal plants across communities in Uganda based on electronic data. Evidence-Based Complementary and Alternative Medicine, ID 3057180. https://doi. org/10.1155/2019/3057180

Ong H. G., Ling S. M., Win T. T. M., Kang D.-H., Lee J.-H., Kim Y.-D., 2018. Ethnobotany of wild medicinal plants used by the Müün ethnic people: A quantitative survey in southern Chin State, Myanmar. Journal of Herbal Medicine, 13: 91-96. https://doi.org/10.1016/j.hermed.2017.09.006

Raj A. J., Biswakam S., Pala N. A., Shukla G., Vineeta, Kumar M., Chakravarty S., et al., 2018. Indigenous uses of ethnomedicinal plants among forest-dependent communities of Northern Bengal, India. Journal of Ethnobiology and Ethnomedicine, 14: 8. https://doi.org/10.1186/s13002018-0208-9

Saotoing P., Vroumsia T., Tchobsala, Tchuenguem F. F.-N., Njan N. A.-M., Messi J., 2011. Medicinal plants used in traditional treatment of malaria in Cameroon. Journal of Ecology and the Natural Environment, 3 (3): 104-117. https://academicjournals.org/journal//ENE/article-abstract/5E8F71C6080

Silva J. R. A., Ramos A. S., Machado M., Moura D. F., Neto Z., Canto-Cavalheiro M. M., et al., 2011. A review of antimalarial plants used in traditional medicine in communities in Portuguese-speaking countries: Brazil, Mozambique, Cape Verde, Guinea-Bissau, São Tomé and Príncipe and Angola. Memórias do Instituto Oswaldo Cruz, 106 (1): 142-158. https:// doi.org/10.1590/S0074-02762011000900019

Swana E. K., Yav T. I., Ngwej L. M., Mupemba B. N., Suprianto, Mukeng C. K., et al., 2018. School-based malaria prevalence: informative systematic surveillance measure to assess epidemiological impact of malaria control interventions in Democratic Republic of the Congo. Malaria Journal, 17: 141. https://doi.org/10.1186/s12936-018-2297-2

Tabuti J. R. S., Lye K. A., Dhillion S. S., 2003. Traditional herbal drugs of Bulamogi, Uganda: plants, use and administration. Journal of Ethnopharmacology, 88: 19-44. https://doi.org/10.1016/S0378-8741(03)00161-2 Tabuti J. R. S., 2008. Herbal medicines used in the treatment of malaria in Budiope county, Uganda. Journal of Ethnopharmacology, 116: 33-42. https://doi.org/10.1016/i.jep.2007.10.036

Taek M. M., Ew B. P., Agil M., 2018. Ethnomedicinal plants used for the treatment of malaria in Malaka, west Timor. Journal of Young Pharmacists, 10 (2): 187-192. https://www.jyoungpharm.org/sites/default/ files/JYoungPharm_10_2 187 0.pdf

Terashima H., Ichikawa M., 2003. A comparative ethnobotany of the Mbuti and Efe Hunter-gatherers in the Ituri forest, Democratic Republic of Congo. African Study Monographs, 24 (1-2): 1-168. https://core.ac.uk/ download/pdf/39201366.pdf

Titanji V. P. K., Zofou D., Ngemenya M., 2008. The antimalarial potential of medicinal plants used for the treatment of malaria in Cameroonian folk medicine. African Journal of Traditional, Complementary and Alternative Medicines, 5 (3): 302-321. https://www.ncbi.nlm.nih.gov/pmc/ articles/PMC2816552/
Tor-anyiin T. A., Sha'ato R., Oluma H. O. A., 2003. Ethnobotanical survey of anti-malarial medicinal plants amongst the Tiv people of Nigeria. Journal of Herbs, Spices \& Medicinal Plants, 10 (3): 61-74. https://doi. org/10.1300/J044v10n03_07

Traore M. S., Baldé M. A., Diallo M. S. T., Baldé E. S., Diané S., Camara A., et al., 2013. Ethnobotanical survey on medicinal plants used by Guinean traditional healers in the treatment of malaria. Journal of Ethnopharmacology, 150: 1145-1153. https://doi.org/10.1016/j.jep.2013.10.048

Umair M., Altaf M., Bussmann R. W., Abbasi A. M., 2019. Ethnomedicinal uses of local flora in Chenab riverine area, Punjab province, Pakistan. Journal of Ethnobiology and Ethnomedicine, 15: 7. https://doi. org/10.1186/s13002-019-0285-4

UNESCO, 2010. Le Patrimoine mondial dans le Bassin du Congo. Centre du Patrimoine mondial de l'UNESCO, 64 p. https://whc.unesco.org/fr/ activites/628/

Vijayakumar S., Yabesh J. E. M., Prabhu S., Manikandan R., Muralidharan B., 2015. Quantitative ethnomedicinal study of plants used in the Nelliyampathy hills of Kerala, India. Journal of Ethnopharmacology, 161: 238-254. https://doi.org/10.1016/i.jep.2014.12.006

WHO, 2019. World Malaria Report 2019. World Health Organization, 232 p. https://www.who.int/publications/i/item/9789241565721

Yetein M. H., Houessou L. G., Lougbégnon T. O., Teka O., Tente B., 2013. Ethnobotanical study of medicinal plants used for the treatment of malaria in plateau of Allada, Benin (West Africa). Journal of Ethnopharmacology, 146: 154-163. https://doi.org/10.1016/i.jep.2012.12.022

Zarei L., Naji-Haddadi S., Pourjabali M., Naghdi N., Tasbih-Forosh M., Shahsavari S., 2017. Systematic review of anti-Rheumatic Medicinal plants: An overview of the effectiveness of articular tissues and joint pain associated with Rheumatoid arthritis. Journal of Pharmaceutical Sciences and Research, 9 (5): 547-551. https://www.jpsr.pharmainfo. in/Documents/Volumes/vol91ssue05/ipsr09051706.pdf

\begin{tabular}{l|l|}
$\begin{array}{l}\text { Rusaati et al. - Authors' contributions } \\
\text { Contributor role }\end{array}$ & Contributor names \\
\hline Data curation & B. I. W. Rusaati, J. W. Kang \\
\hline Formal Analysis & J. W. Kang \\
\hline \begin{tabular}{l} 
Investigation \\
\hline Methodology
\end{tabular} & B. I. W. Rusaati \\
\hline Resources & B. I. W. Rusaati \\
\hline Supervision & B. I. W. Rusaati, A. P. Gendusa \\
\hline \begin{tabular}{l} 
validation \\
\hline Visualisation
\end{tabular} & J. W. Park, S. H. Joo \\
\hline $\begin{array}{l}\text { Writing- original draft } \\
\text { preparation }\end{array}$ & J. W. Kang \\
\hline $\begin{array}{l}\text { Writing- Review } \\
\text { and Editing }\end{array}$ & J. W. Kang, N. C. Masumbuko \\
\hline B. I. W. Rusaati \\
\hline
\end{tabular}

Bois et Forêts des Tropiques - Revue scientifique du Cirad (c) Bois et Forêts des Tropiques (c) Cirad
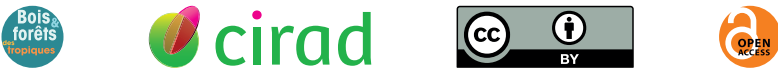

Cirad - Campus international de Baillarguet, 34398 Montpellier Cedex 5, France - Contact : bft@cirad.fr - ISSN : L-0006-579X 